\title{
过渡金属催化的吡啶的官能化
}

\author{
张 斌 $a, b$ 周其忠 $*, b$ 陈仁尔 ${ }^{b}$ 蒋华江 $*, b$ \\ ( ${ }^{a}$ 浙江工业大学药学院 杭州 310014) \\ ( ${ }^{b}$ 台州学院化学系 台州 317000)
}

摘要 吡啶是药物和天然产物的重要骨架, 是医药化工的重要的中间体. 介绍了过渡金属催化的吡啶在 2 位、3 位或 4 位发生的烷基化、烯基化和芳基化等 $\mathrm{C}-\mathrm{H}$ 键活化反应，还概括了吡啶的官能化在药物和天然产物合成中的应用.

关键词 吡啶; 官能化; 烷基化; 烯基化; 芳基化

\section{Transition-Metal-Catalyzed Functionalization of Pyridines}

\author{
Zhang, Bin ${ }^{a, b}$ Zhou, Qizhong* ${ }^{*, b}$ Chen, Rener ${ }^{\mathrm{b}} \quad$ Jiang, Huajiang ${ }^{*, b}$ \\ ( ${ }^{a}$ College of Pharmacy, Zhejiang University of Technology, Hangzhou 310014) \\ ( ${ }^{b}$ Department of Chemistry, Taizhou University, Taizhou 317000)
}

\begin{abstract}
Pyridines are key backbones of drugs and natural products as well as the key intermediates of medicinal engineering. This paper summarizes transition-metal-catalyzed functionalization of pyridines via $\mathrm{C}-\mathrm{H}$ activation such as alkylation, alkenylation and arylation etc. as well as their utilities in synthesis of drugs and natural products.

Keywords pyridines; functionalization; alkylation; alkenylation; arylation
\end{abstract}

吡啶是一类数量很大的重要的一种杂环化合物, 是 重要的化工原料. 吡啶衍生物可以作为配体, 来催化有 机反应. 吡啶是药物分子的重要骨架, 如 Dimebolin 是 治疗阿尔茨海默病的新药, Altinicline 用于治疗帕金森 病(Scheme 1). 吡啶也是具有重要生理活性分子的骨架 (Scheme 2) $^{[1,2]}$.
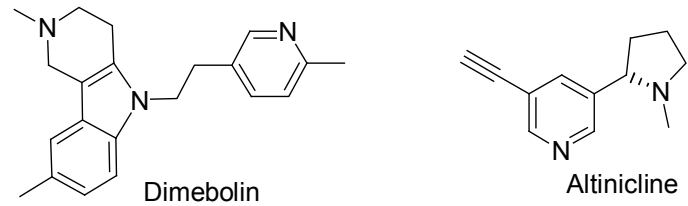

Scheme 1

吡啶能通过开环反应生成 Zinke aldehyde, 是合成 其它分子的重要中间体(Scheme 3) $)^{[3,4]}$.

吡啶环具有反应活性, 在过渡金属钯、铑、钓、镍 等催化下, 在吡啶的 2 位、3 位和 4 位上可以发生 C-H 键活化反应，生成烷基、烯基、芳基乃至羰基取代的吡 啶. 这对于合成药物分子, 具有重要生理活性的分子,

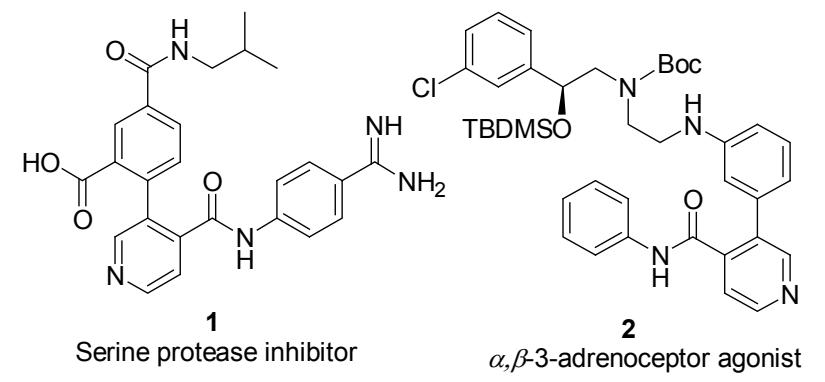<smiles>CCCCCCCCCCCC(O)C=Cc1cccc(C2OC2CCCCO)n1</smiles><smiles>CCc1ccc(CCOc2ccc(CC3SC(=O)NC3=O)cc2)nc1</smiles>

Scheme 2

天然产物分子具有重要的意义.

\footnotetext{
*E-mail: qizhongchou@yahoo.com; jhj@tzc.edu.cn

Received January 29, 2012; revised April 6, 2012, 2012; published online April 24, 2012.

Project supported by the National Natural Science Foundation of China (No. 21172166) and the Natural Science Foundation of Zhejiang Province (No. Y4100783)

国家自然科学基金(No. 21172166)和浙江省自然科学基金(No. Y4100783)资助项目.
} 


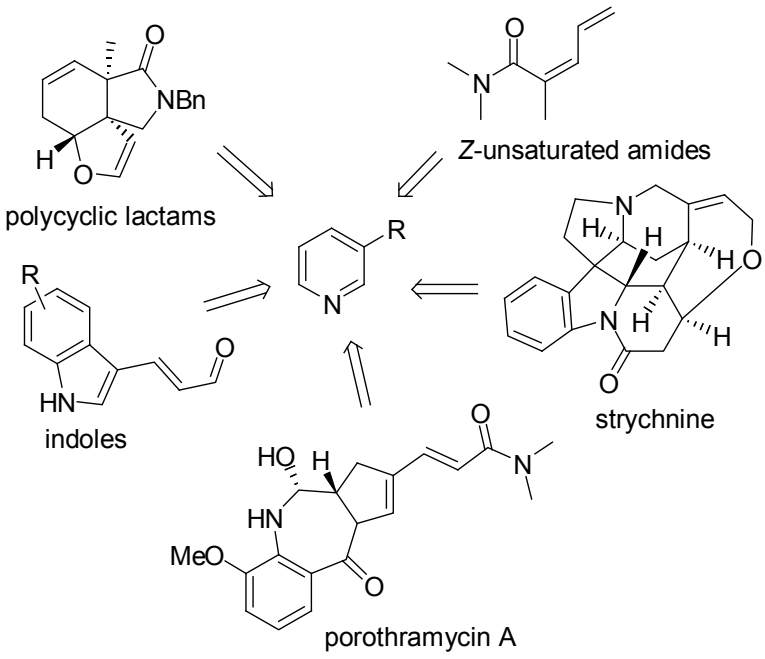

Scheme 3

2011 年日本的 $\mathrm{Nakao}^{[5]}$ 综述了过渡金属催化的 $\mathrm{C}$ $\mathrm{H}$ 键的官能化用于取代吡啶的合成, 这篇综述引用的参 考文献较少, 未能全面反应该领域的发展. 本文较为全 面地综述了自 1985 年以来该领域的发展状况, 介绍了 过渡金属钯、铑、钉、镍等催化下在吡啶的 2 位、3 位 和 4 位上发生的官能化反应及其在药物和天然产物合成 中的应用.

\section{1 吡啶 2 位上发生的官能化反应}

\section{1 吡啶 2 位上发生的烷基化反应}

1985 年美国的 Laine 等 ${ }^{[6]}$ 报道了 $\mathrm{Ru}_{3}(\mathrm{CO})_{12}$ 和 $\mathrm{Os}_{3}(\mathrm{CO})_{12}$ 脱去 $\mathrm{CO}$ 后能与吡啶生成金属络合物, 在该金 属络合物中, 吡啶 2 位的碳与金属成键(Eq. 1). 这是最 先报道的吡啶 2 位上的 $\mathrm{C}-\mathrm{H}$ 键被过渡金属络合物活化 的例子, 为吡啶的官能化反应提供了先例和理论基础.

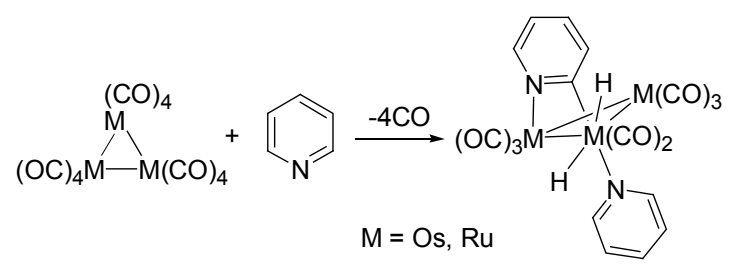

1989年美国爱荷华大学的 Jordan 等 ${ }^{[7]}$ 报道了锆催化 的 2-甲基吡啶与丙烯的偶联反应(Eq. 2).

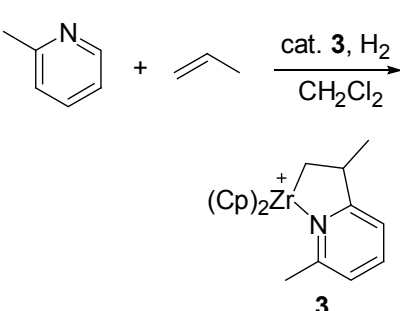<smiles>Cc1cccc(C(C)C)n1</smiles>

其反应机理是 $\mathbf{3}$ 加氢生成 $\mathbf{A}, \mathbf{A}$ 与 2-甲基吡啶发生 配体交换生成产物和 $\mathbf{B}, \mathbf{B}$ 脱氢生成 $\mathbf{C}, \mathbf{C}$ 与丙烯反应生 成 3, 完成了催化循环(Scheme 4).

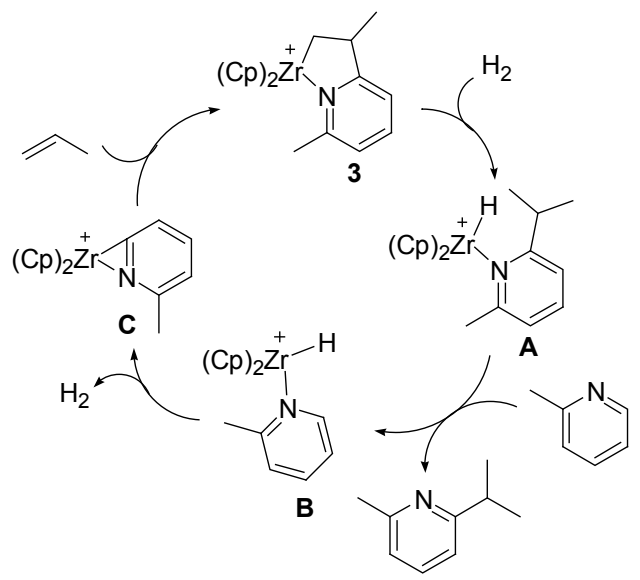

Scheme 4

1994 年 Jordan 等 ${ }^{[8]}$ 报道了手性锆催化的吡啶 2 位上 的对映选择性烯烃的插入，获得了 $58 \%$ ee (Eq. 3). 该反 应机理与 Scheme 4 相似.

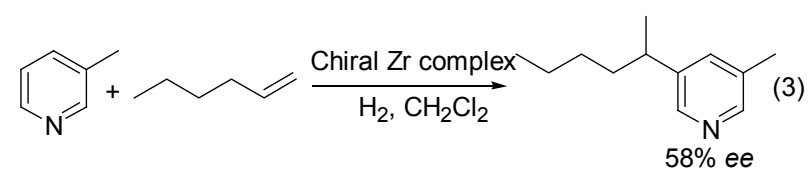

2007 年美国加州大学伯克利分校的 Ellman 和 Bergman 等 ${ }^{[9]}$ 报道了 $\left[\mathrm{RhCl}(\mathrm{coe})_{2}\right]_{2}$ 催化的吡啶 2 位上的 直链烷基化，产率 50\% 83\% (Eq. 4).

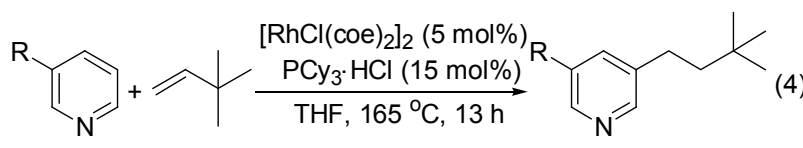<smiles>Cc1cncc(CCC(C)(C)CC(C)c2cncc(CCC(C)(C)C(F)(F)F)c2)c1</smiles>

2010 年 Ellman 和 Bergman 等 ${ }^{[10]}$ 报道了 $[\mathrm{RhCl}(\mathrm{cod})]_{2}$ 催化的吡啶 2 位上的分子内的烷基化，产率为 50\% $96 \%$ (Eq. 5).

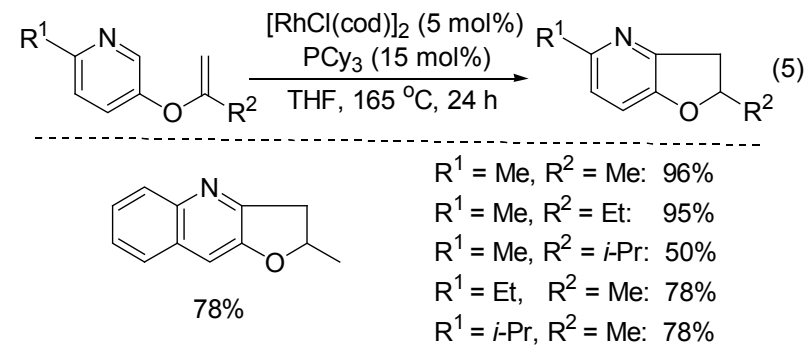


2009 年加拿大麦吉尔大学的李朝军等 ${ }^{[11]}$ 报道了 $\mathrm{Sc}(\mathrm{OTf})_{3}$ 催化的吡啶与环辛烷的烷基化，吡啶的两个邻 位均可发生烷基化, 52\% 71\%总产率(Eq. 6).

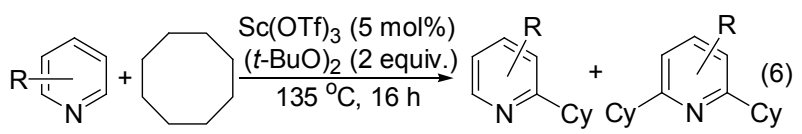
Cy-H (15.5 equiv.) A B<smiles>Clc1cc(C2CCCCC2)nc2ccccc12</smiles><smiles>[Y6]c1nccc2ccccc12</smiles>

$\mathrm{R}=\mathrm{H}:$ $\mathrm{R}=4-\mathrm{Me}:$ $64 \%(\mathbf{A}: \mathbf{B}=1: 1.3)$ $68 \%(A: B=1: 1)$ $\mathrm{R}=4-\mathrm{Ph}: \quad 71 \%(\mathbf{A}: \mathbf{B}=1: 1.5)$ $72 \%$ $74 \%$ $\mathrm{R}=4-\mathrm{CO}_{2}$ Me: $52 \%$ (only A) $\mathrm{R}=2-\mathrm{Me}: \quad 53 \%$ (only $\mathbf{A}$ ) $\mathrm{R}=2-\mathrm{Ph}: \quad 52 \%$ (only $\mathbf{A}$ )

2011 年日本理化学研究所的侯召民等 ${ }^{[12]}$ 报道了过 渡金属锌催化的吡啶的支链烷基化，产率 86\% 99\%; 过渡金属钇催化苯乙烯对吡啶的直链烷基化, 产率 90\% 99\% (Scheme 5).

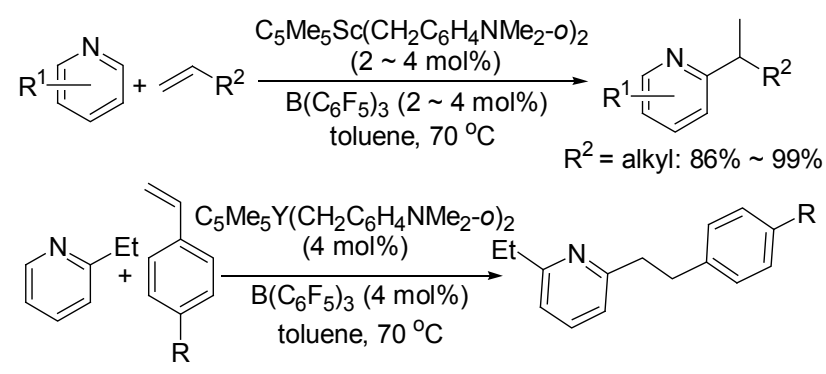

$$
\begin{array}{lll}
\mathrm{R}=\mathrm{H}: & 94 \% ; & \mathrm{R}=\mathrm{Me}: \quad 99 \% \\
\mathrm{R}=t-\mathrm{Bu}: & 98 \% ; \quad \mathrm{R}=\mathrm{F}: \quad 90 \% \\
\mathrm{R}=\mathrm{Cl}: & 94 \% ; & \mathrm{R}=\text { OMe: } 96 \%
\end{array}
$$

\section{Scheme 5}

锌和钎催化的反应机理如 Scheme 6 所示 ${ }^{[12]}$. 配体 $\mathbf{A}$ 和吡啶作用脱去一分子烷烃生成 $\mathbf{B}$, 烷基取代的烯烃 对 $\mathbf{B}$ 插入生成 $\mathbf{C}, \mathbf{C}$ 和吡定发生配体交换生成产物 $\mathbf{6}$ 和 $\mathbf{B}$, 完成了催化循环. 苯乙烯对 $\mathbf{B}$ 插入生成 $\mathbf{D}, \mathbf{D}$ 和吡定 发生配体交换生成产物 $\mathbf{5}$ 和 $\mathbf{B}$, 完成了催化循环.

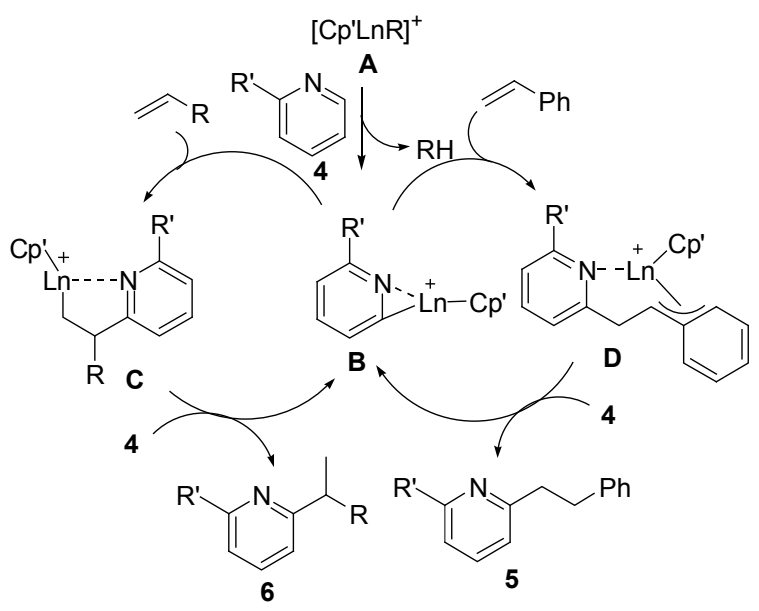

Scheme 6

\section{2 吡啶 2 位上发生的烯基化反应}

2003 年日本京都大学的 Murakami 等 ${ }^{[13]}$ 报道了 $\mathrm{CpRu}\left(\mathrm{PPh}_{3}\right)_{2} \mathrm{Cl}$ 催化的吡啶和炔基硅反应生成反式 2-烯 基吡定，产率 $0 \sim 90 \%$, 反应具有区域选择性和立体选 择性(Eq. 7).

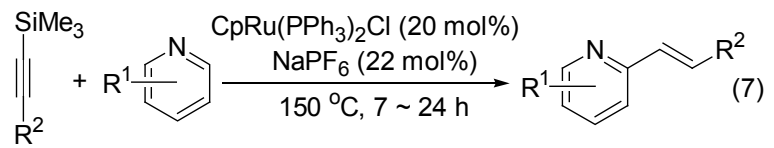

$$
\begin{aligned}
& \mathrm{R}^{1}=\mathrm{H}, \quad \mathrm{R}^{2}=\mathrm{Ph}: \quad 87 \% \\
& \mathrm{R}^{1}=\mathrm{H}, \quad \mathrm{R}^{2}=p-\mathrm{Me}_{-}-\mathrm{C}_{6} \mathrm{H}_{4}: \quad 92 \% \\
& \mathrm{R}^{1}=\mathrm{H}, \quad \mathrm{R}^{2}=n-\mathrm{C}_{5} \mathrm{H}_{11}: \quad 90 \% \\
& \mathrm{R}^{1}=\mathrm{H}, \quad \mathrm{R}^{2}=1 \text {-cyclohexenyl: } 75 \% \\
& \mathrm{R}^{1}=2-\mathrm{Me}, \quad \mathrm{R}^{2}=\mathrm{Ph}: \quad 0 \\
& \mathrm{R}^{1}=3-\mathrm{Me}, \quad \mathrm{R}^{2}=\mathrm{Ph}: \quad 68 \% \\
& \mathrm{R}^{1}=4-\mathrm{Me}, \quad \mathrm{R}^{2}=\mathrm{Ph}: \quad 76 \% \\
& \mathrm{R}^{1}=4-\mathrm{Et}, \quad \mathrm{R}^{2}=\mathrm{Ph}: \quad 66 \% \\
& \mathrm{R}^{1}=4-\mathrm{OMe}, \mathrm{R}^{2}=\mathrm{Ph}: \quad 74 \%
\end{aligned}
$$

该反应机理是: $\mathrm{CpRu}\left(\mathrm{PPh}_{3}\right)_{2} \mathrm{Cl}$ 与炔基硅生成离子 型的亚乙烯基钉 $\mathbf{A}, \mathbf{A}$ 与吡啶配位生成 $\mathbf{B}, \mathbf{B}$ 发生分子内 的 $2+2$ 环加成生成 $\mathbf{C}, \mathbf{C}$ 在吡啶的作用下脱保护生成 D, D 质子化然后异构化生成反式的 2-烯基吡啶(Scheme 7) ${ }^{[13]}$.

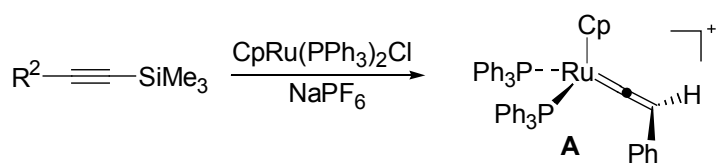

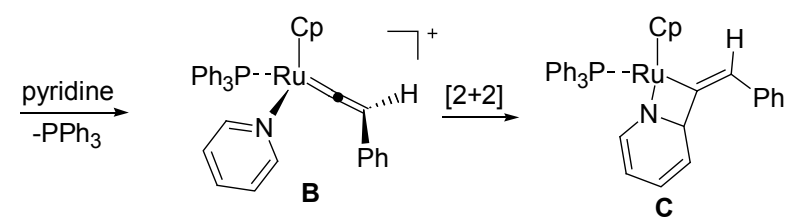

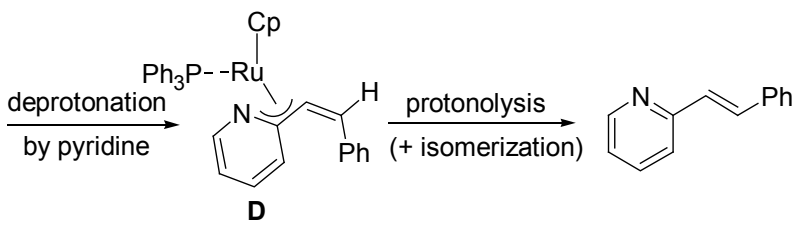

\section{Scheme 7}

2007 年日本京都大学的 Nakao 和 Hiyama 等 ${ }^{[14]}$ 报道 了 $\mathrm{Ni}(\operatorname{cod})_{2}$ 催化的氧化吡啶对炔烃的加成反应, 高度区 域选择性和立体选择性地生成反式 2-烯基氧化吡啶, 54\% 81\%产率(Eq. 8).

该反应机理是: 零价镍与炔烃配位形成 $\mathbf{A}, \mathbf{A}$ 与氧 化吡啶的 2 位 $\mathrm{C}-\mathrm{H}$ 键氧化加成生成吡啶氢化镍 $\mathbf{B}$, 吡 啶氢化镍 $\mathbf{B}$ 对炔烃顺式加成生成 $\mathbf{C}, \mathbf{C}$ 发生还原消除生 成反式 2-烯基氧化吡啶(Scheme 8). 


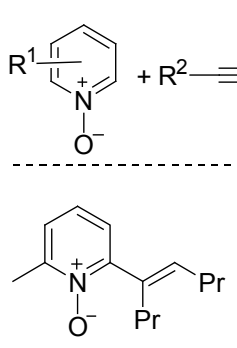

$67 \%, E: Z=93: 7$
$\mathrm{Ni}(\operatorname{cod})_{2}(10 \mathrm{~mol} \%)$ $\underset{\text { toluene, } 35^{\circ} \mathrm{C}}{\stackrel{\mathrm{PCyp}_{3}(10 \mathrm{~mol} \%)}{\longrightarrow}} \mathrm{R}$<smiles>CCCC=C(CCC)c1ccc(C)c(C)[n+]1[O-]</smiles>
$59 \%, E: Z=97: 3$ $\mathrm{O}^{-} \mathrm{R}^{2}$<smiles>CCCC(=CP)c1cc(C)cc(C)[n+]1[O-]</smiles>

$54 \%, E: Z=94: 6$<smiles>CCCC=C(P)c1c(C)ccc(C)[n+]1[O-]</smiles>

$66 \%, E: Z=>99: 1$

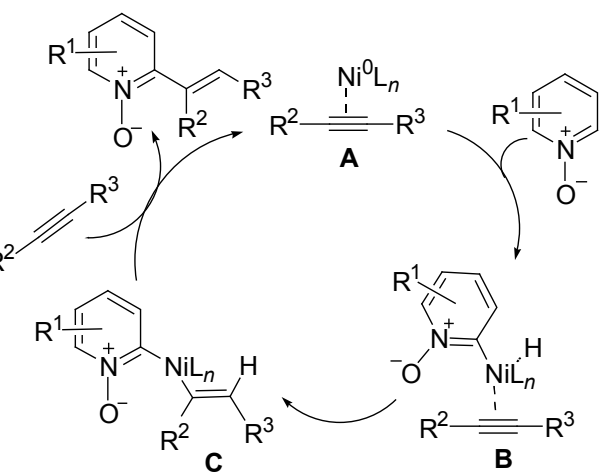

Scheme 8

2008 年 Nakao 和 Hiyama 等 ${ }^{[15]}$ 报道了 $\mathrm{Ni}(\mathrm{cod})_{2}$ 和 LA 催化的吡定对炔烃的加成反应, 高度区域选择性和 立体选择性地生成反式 2-烯基吡啶，30\%～91\%产率 (Eq. 9).
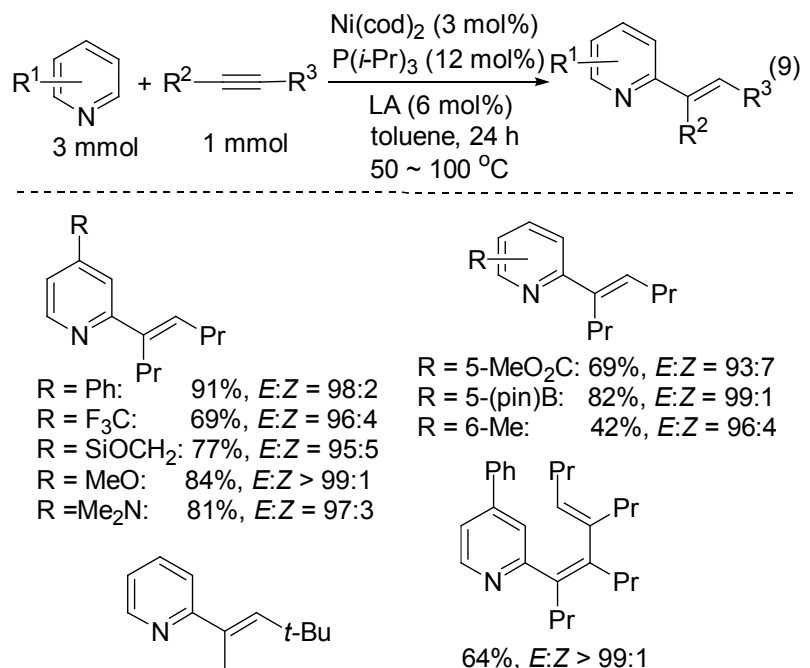

$87 \%, E: Z>99: 1$<smiles>C=C(C(=O)OC)c1ccccn1</smiles>

$56 \%, E: Z=63: 37$

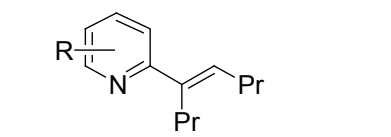

$\mathrm{R}=5-\mathrm{MeO}_{2} \mathrm{C}: 69 \%, E: Z=93: 7$ $\mathrm{R}=5-($ pin)B: $\quad 82 \%, E: Z=99: 1$ $\mathrm{R}=6-\mathrm{Me}: \quad 42 \%, E: Z=96: 4$<smiles>CC=C(CCCC)C(CCC)=C(CC)c1cc(-c2ccccc2)ccn1</smiles>

$64 \%, E: Z>99: 1$

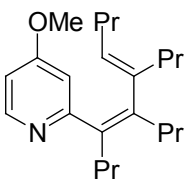

$46 \%, E: Z>99: 1$
该反应机理是: 零价镍与炔烃配位形成 $\mathbf{A}, \mathbf{A}$ 与吡 啶的 2 位 $\mathrm{C}-\mathrm{H}$ 键氧化加成生成吡啶氢化镍 $\mathbf{B}$, 吡啶氢 化镍 $\mathbf{B}$ 对炔烃顺式加成生成 $\mathbf{C}$ 或 $\mathbf{D}, \mathbf{C}$ 发生还原消除生 成反式 2-烯基吡定. D 发生还原消除生成另外的产物 (Scheme 9).

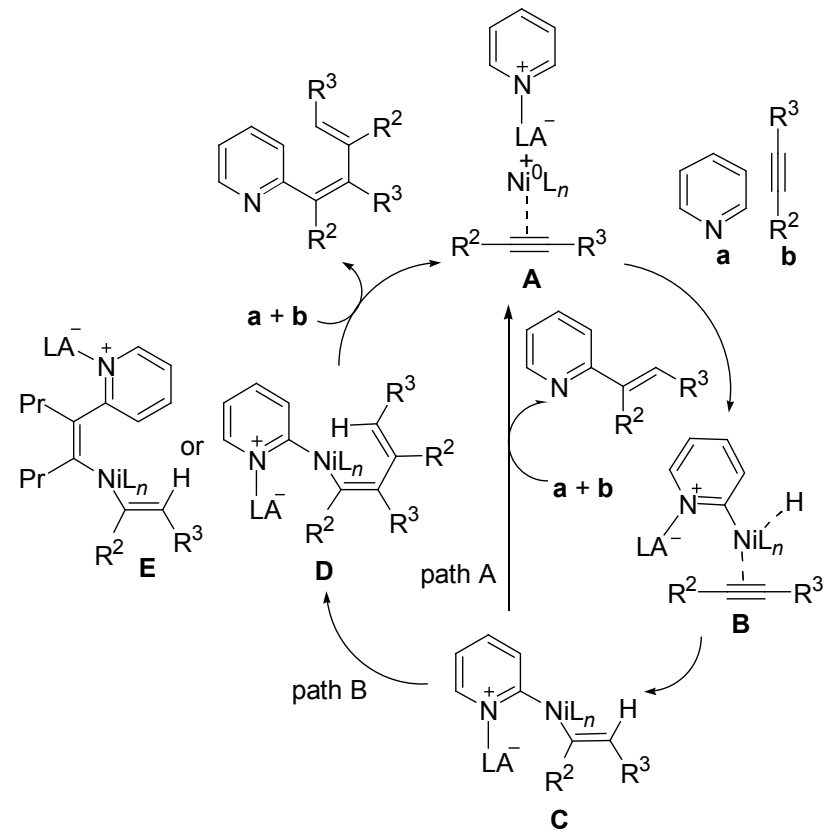

Scheme 9

2008 年韩国先进科学技术研究所的 Chang 等 ${ }^{[16]}$ 报 道了 $\mathrm{Pd}(\mathrm{OAc})_{2}$ 催化的氧化吡定与烯烃的偶联反应，高 度区域选择性和立体选择性地生成反式 2-烯基氧化吡 啶, 53\% 91\%产率(Eq. 10).

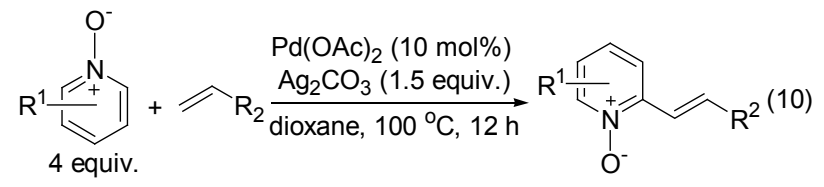<smiles>CC(C)(C)OC(=O)C=Cc1cnc2ccccc2[n+]1[O-]</smiles>

$$
\begin{array}{llr}
\mathrm{R}^{1}=\mathrm{H}, & \mathrm{R}^{2}=\mathrm{CO}_{2} t-\mathrm{Bu}: & 91 \% \\
\mathrm{R}^{1}=\mathrm{H}, & \mathrm{R}^{2}=\mathrm{CONMe}_{2}: & 87 \% \\
\mathrm{R}^{1}=\mathrm{H}, & \mathrm{R}^{2}=\mathrm{COMe}: & 62 \% \\
\mathrm{R}^{1}=\mathrm{H}, & \mathrm{R}^{2}=\mathrm{PO}(\mathrm{OEt})_{2}: & 70 \% \\
\mathrm{R}^{1}=\mathrm{H}, & \mathrm{R}^{2}=t-\mathrm{Bu}: & 53 \% \\
\mathrm{R}^{1}=\mathrm{H}, & \mathrm{R}^{2}=\mathrm{Ph}: & 64 \% \\
\mathrm{R}^{1}=4-\mathrm{Ph}, \mathrm{R}^{2}=\mathrm{CO}_{2} t-\mathrm{Bu}: & 68 \% \\
\mathrm{R}^{1}=6-\mathrm{Ph}, \mathrm{R}^{2}=\mathrm{CO}_{2} t-\mathrm{Bu}: & 73 \% \\
\mathrm{R}^{1}=5-\mathrm{Ph}, \mathrm{R}^{2}=\mathrm{CO}_{2} t-\mathrm{Bu}: & 88 \%
\end{array}
$$

该反应机理是: $\mathrm{Pd}(\mathrm{OAc})_{2}$ 与氧化吡啶 2 位 $\mathrm{C}-\mathrm{H}$ 键 发生氧化加成生成 $\mathbf{A}$ (氧化吡啶碳氢键的活化), $\mathbf{A}$ 对烯 烃插入生成 $\mathbf{B}, \mathbf{B}$ 发生还原消除得到产物和零价钯, $\mathrm{Ag}_{2} \mathrm{CO}_{3}$ 将零价钯氧化成二价钯, 完成了催化循环 (Scheme 10). 


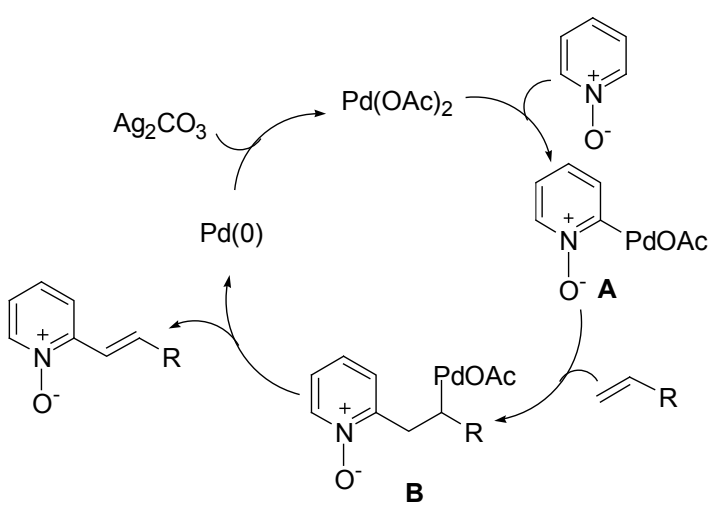

Scheme 10

2009 年郑州大学的崔秀灵和吴养杰等 ${ }^{[17]}$ 报道了 $\mathrm{Pd}(\mathrm{OAc})_{2}$ 催化的氧化喹啉与烯烃的偶联反应, 高度区域 选择性和立体选择性地生成反式 2-烯基氧化喹啉, $27 \% \sim 95 \%$ 产率(Eq. 11). 该反应机理与 Scheme 10 所述 的钯催化机理类似.

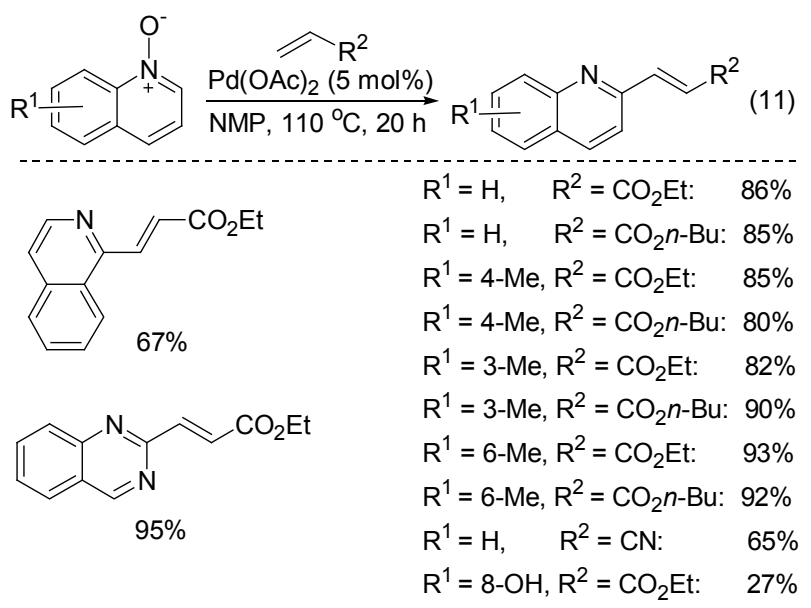

2010 年加拿大蒙特利尔大学的 Charette 等 ${ }^{[2]}$ 报道了 吡啶叶立德和烯基碘在 $\mathrm{CuBr}_{2}$ 催化下发生交叉偶联反 应，产率 30\% 93\% (Eq. 12).

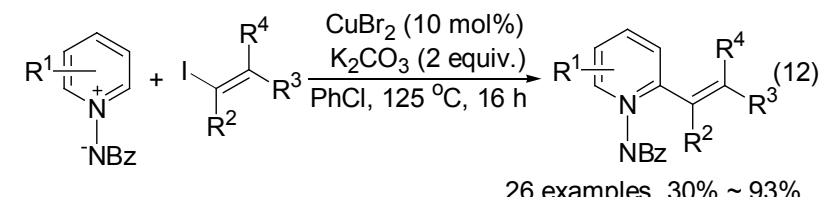
26 examples, $30 \% \sim 93 \%$

该反应机理如 Scheme 11 所示: 两价铜被吡啶叶立 德还原为一价铜, 一价铜与吡啶叶立德通过碳氢键活化 配位生成 $\mathbf{A}, \mathbf{A}$ 与烯基碘氧化加成生成 $\mathbf{B}, \mathbf{B}$ 发生还原消 除生成产物和一价铜, 完成的催化循环.

2011 年中国科学院大连化学物理研究所的李兴伟 等 ${ }^{[18]}$ 报道了铑催化的吡啶的 2,3 位上的并环反应(Eq. 13).

反应机理如 Scheme 12 所示: 4-甲胺酰基吡啶与

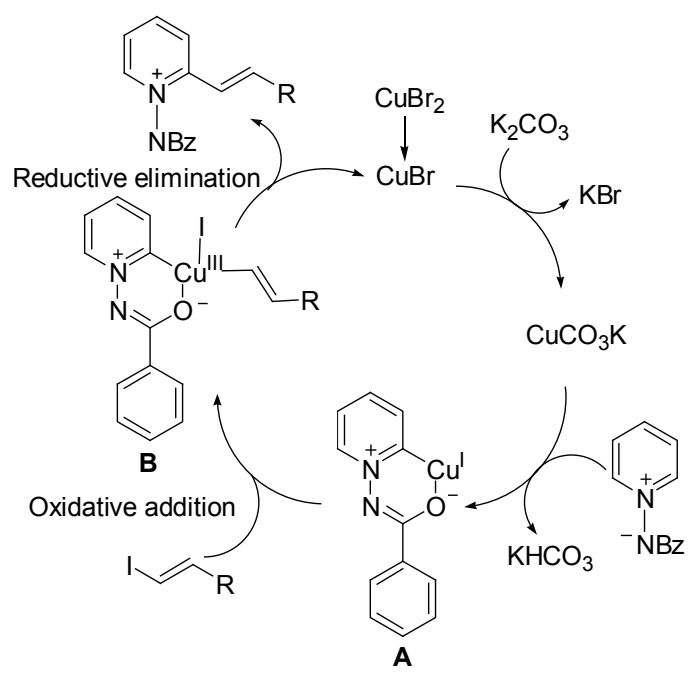

Scheme 11

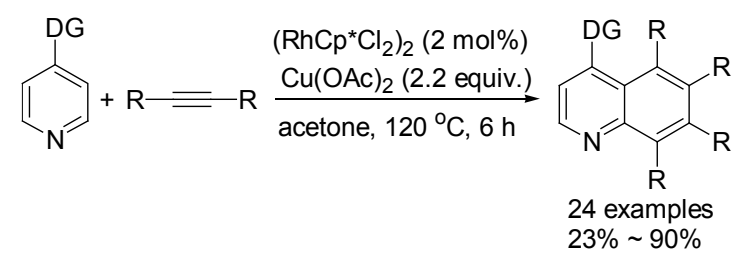

$\mathrm{RhCp}^{*}(\mathrm{OAc})_{2}$ 反应脱去一分子醋酸生成 $\mathbf{B}, \mathbf{B}$ 分子内脱 去一分子醋酸得到 $\mathrm{C}, \mathrm{C}$ 与二苯基乙炔配位得到 $\mathrm{D}, \mathrm{D}$ 发 生二苯乙炔的分子内插入得到 $\mathbf{E}, \mathbf{E}$ 和醋酸反应得到 $\mathbf{F}, \mathbf{F}$ 分子内脱去一分子醋酸得到 $\mathbf{G}, \mathbf{G}$ 与与二苯基乙炔 配位得到 $\mathbf{H}, \mathbf{H}$ 发生二苯乙炔的分子内插入得到 I, I 发 生还原消除脱去 $\mathrm{Rh}(\mathrm{I})$ 得到产物 $\mathbf{J}, \mathrm{Rh}(\mathrm{I})$ 经 $\mathrm{Cu}(\mathrm{OAc})_{2}$ 氧 化得到 $\mathrm{RhCp} *(\mathrm{OAc})_{2}$, 完成了催化循环.

\section{3 吡啶 2 位上发生的芳基化反应}

2000 年以色列耶路撒冷希伯莱大学的 Sasson 等 ${ }^{[19]}$ 报道了卤代芳烃和吡啶在锌作用下在钯催化下可发生 还原交叉偶联反应生成 2-苯基吡啶(Eq. 14).

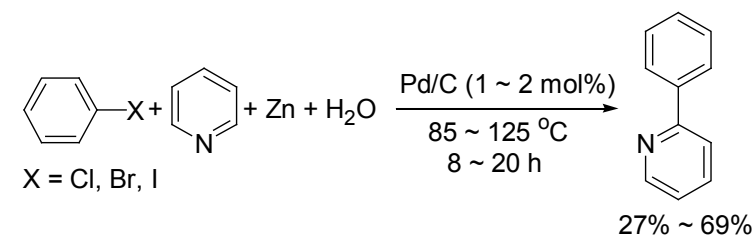

2005 年加拿大渥太华大学的 Fagnou 等 ${ }^{[20]}$ 报道了氧 化吡啶和溴代芳烃在钯催化下可发生交叉偶联反应生 成 2-芳基基吡啶(Eq. 15). 该反应机理和 Scheme 10 所述 的机理类似.

2008 年 Charette 等 ${ }^{[21]}$ 报道了吡定叶立德和芳基溴 在 $\mathrm{Pd}(\mathrm{OAc})_{2}$ 催化下发生交叉偶联反应，产率 $50 \%$ ～ $80 \%$ (Eq. 16). 该反应机理和 Scheme 10 所述的类似. 


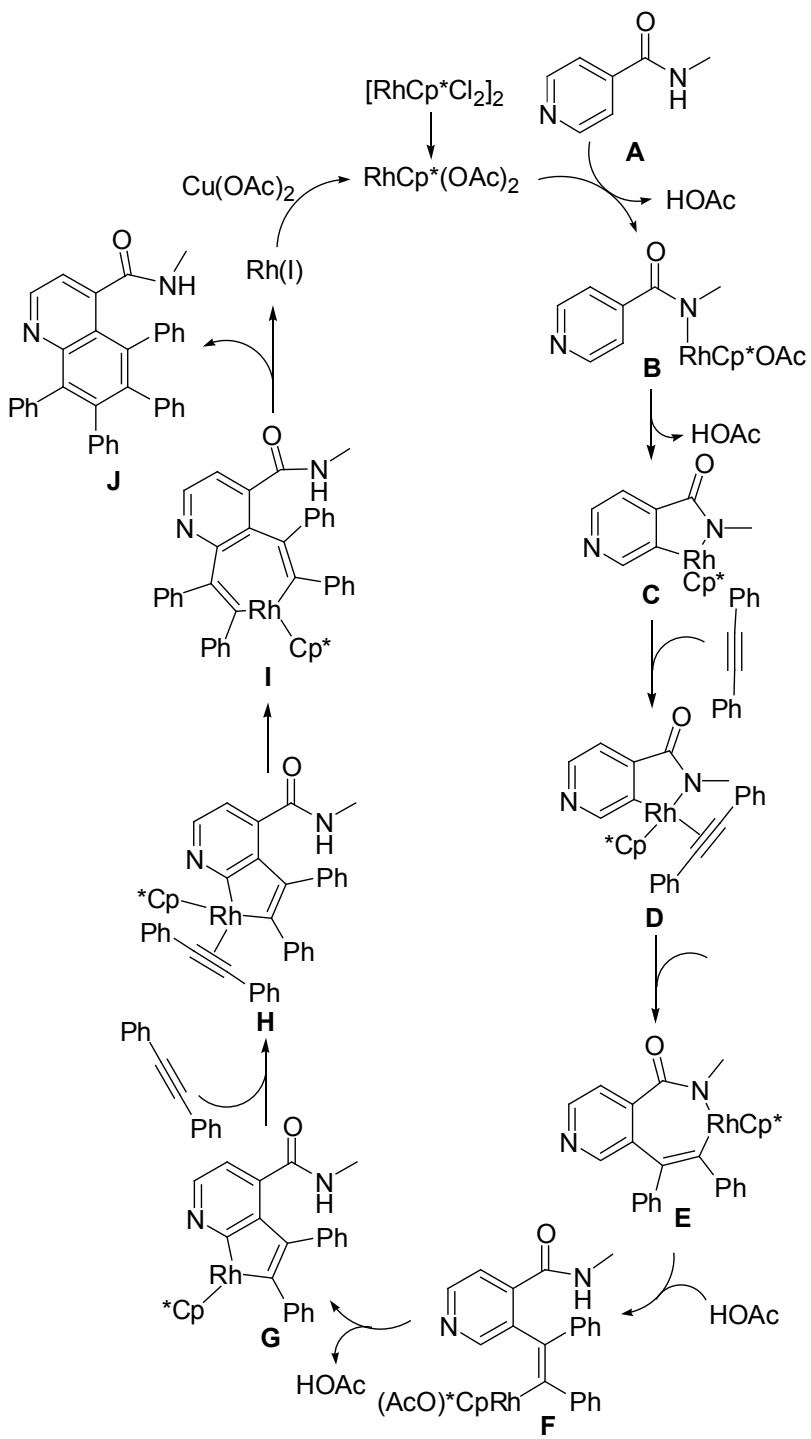

Scheme 12

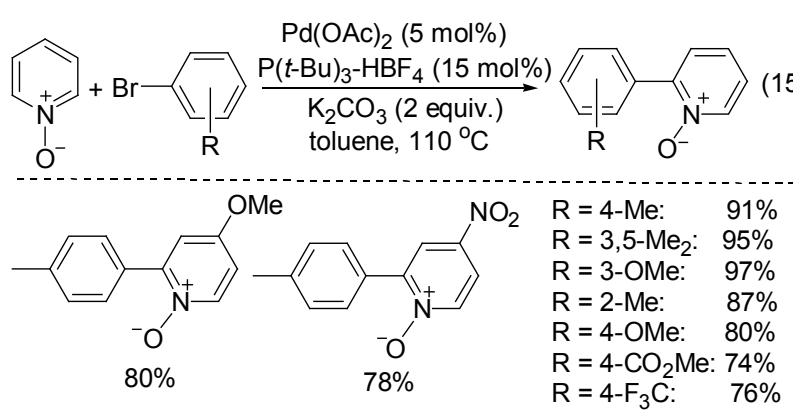

2008 年 Chang 等 ${ }^{[16]}$ 报道了 $\mathrm{Pd}(\mathrm{OAc})_{2}$ 催化的氧化吡 啶与芳烃的偶联反应，高度区域选择性选地生成 2-芳基 氧化吡啶， $42 \% \sim 79 \%$ 产率 (Eq. 17). 该反应机理与 Scheme 10 所述的机理类似.

2010 年, 四川大学的游劲松 ${ }^{[22]}$ 报道了 $\mathrm{Pd}(\mathrm{OAc})_{2}$ 催 化的氧化吡啶与杂环芳烃呋喃和噻酚的偶联反应, 高度 区域选择性选地生成 2-芳基氧化吡啶，60\% 78\%产率 (Eq. 18).
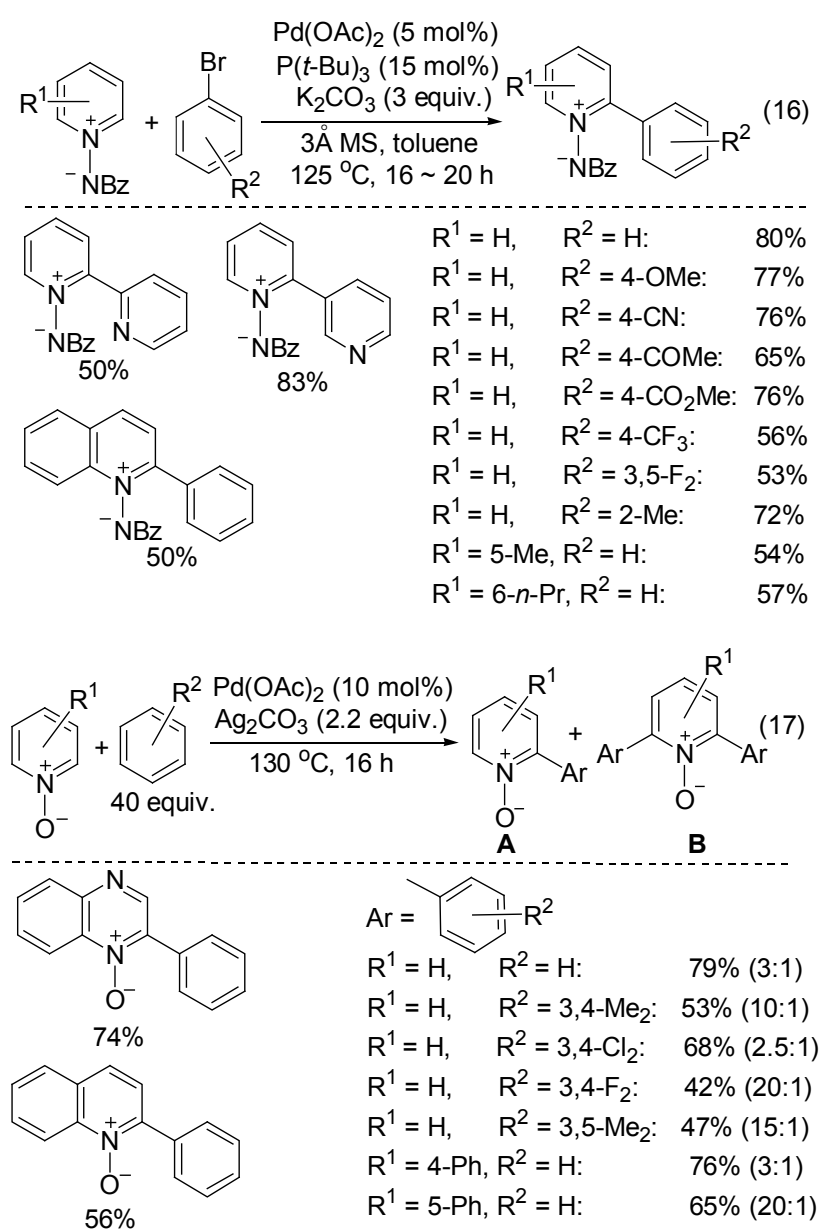

I

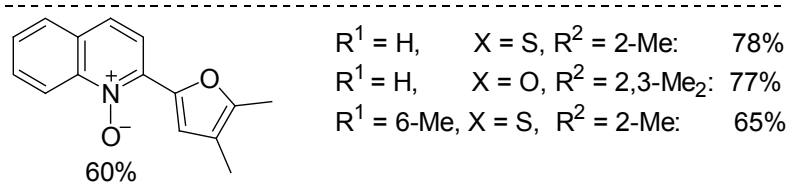

2011 年李兴伟和东北师范大学张宏 ${ }^{[23]}$ 报道了 $\mathrm{Pd}(\mathrm{OAc})_{2}$ 催化的氧化吡啶与杂环芳烃 $N$-取代吡络和 $N$ 取代吲哚的偶联反应，偶联反应发生在氧化吡啶的 2 位 和 $N$-取代吡络或 $N$-取代吲哚的 3 位，45\% 81\%产率 (Eq. 19).

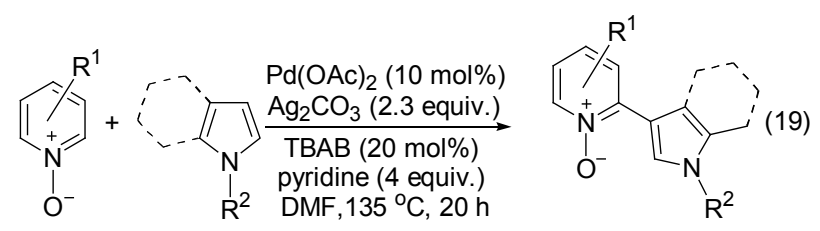

19 examples, $45 \%$ $81 \%$

2011 年德国生物化学与化学研究所的 Tzschucke 等 ${ }^{[24]}$ 报道了氧化吡啶和溴代吡啶在 $\mathrm{Pd}(\mathrm{OAc})_{2}$ 催化下发 生交叉偶联反应，产率 14\%～73\% (Scheme 13). 


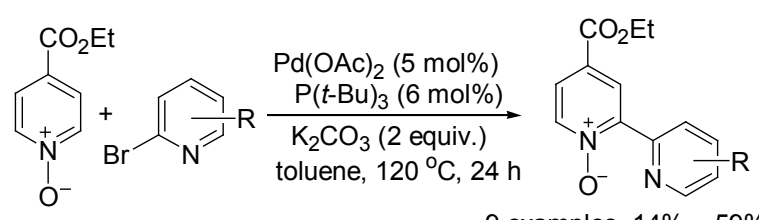

9 examples, $14 \% \sim 59 \%$

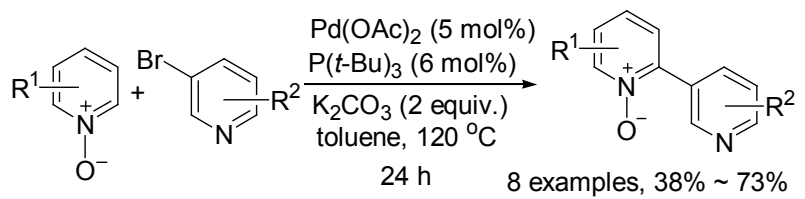

$$
\text { 8 examples, } 27 \% \sim 64 \%
$$

\section{Scheme 13}

2012 年 Hartwig 等 ${ }^{[25]}$ 提出了氧化吡啶 2 位芳基化的 机 理(Scheme 14): $\mathrm{ArBr}$ 对 $\left(\mathrm{P} t-\mathrm{Bu}_{3}\right) \mathrm{Pd}(0)$ 氧化加成生成 $\mathbf{D}, \mathbf{D}$ 与 $\mathbf{B}$ 转金属化生成 $\mathbf{C}$ 和 $\mathbf{A}, \mathbf{C}$ 发生还原消除生成产 物和零价钯完成左边的催化循环. $\mathbf{A}$ 与氧化吡啶反应生 成 B.

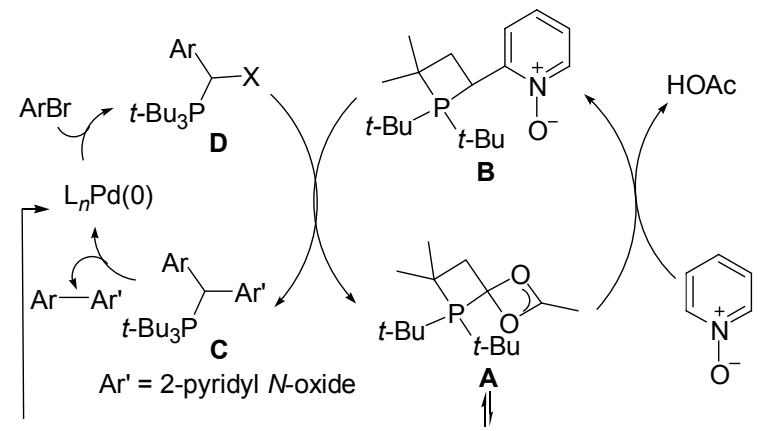

$\mathrm{Pd}(\mathrm{OAc})_{2}$ and $\mathrm{P}(t-\mathrm{Bu})_{3} \longrightarrow 1 / 2\left[\mathrm{Pd}\left(\mu^{2}-\mathrm{OAc}\right)\left(\kappa^{2}-t-\mathrm{Bu}_{2} \mathrm{PCMe}_{2} \mathrm{CH}_{2}\right)\right]_{2}$

\section{Scheme 14}

2009 年, 日本大阪大学的 Tobisu 和 Chatani 等 ${ }^{[26]}$ 报道了在 $\mathrm{Ni}(\operatorname{cod})_{2}$ 催化下, 吡啶和二苯基锌反应生成 $2-$ 苯基吡啶，反应具有区域选择性. 反应机理是二苯基锌 对吡定的 $\mathrm{C}=\mathrm{N}$ 加成, 然后脱去 $\mathrm{ZnH}$ 生成产物(Scheme 15).

2009 年日本佐上化学研究中心 Yamakawa 等 ${ }^{[27]}$ 报 道了吡定在 $\mathrm{NiCp}_{2}$ 催化下和芳基溴生成 $o, m, p$-取代的芳 基吡啶(Eq. 20).

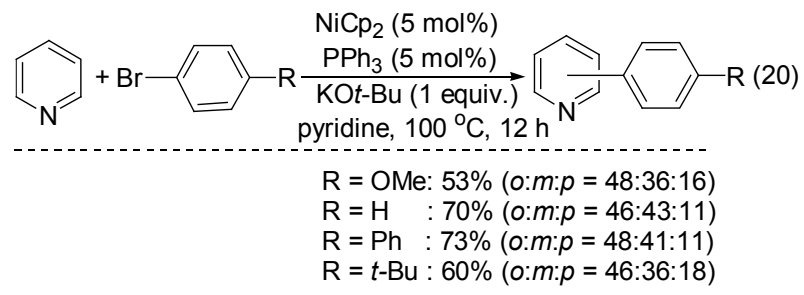

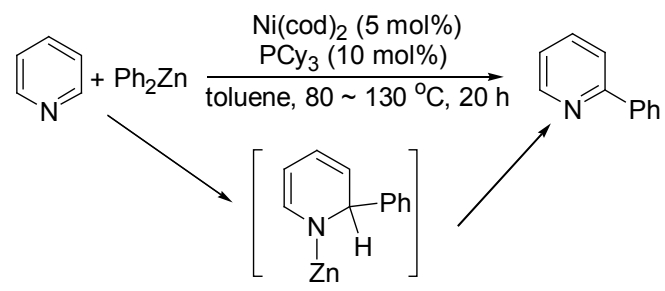

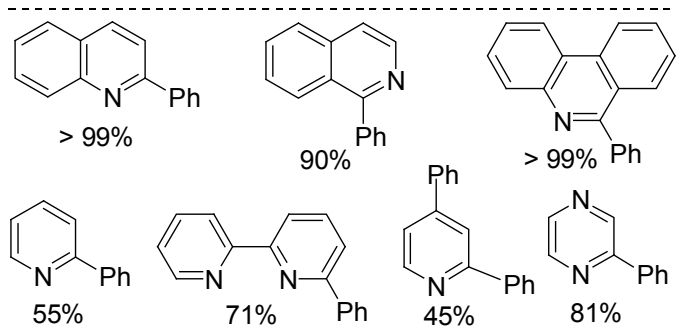

Scheme 15

2008 年 Bergman 和 Ellman 等 ${ }^{[28]}$ 在报道了 $[\mathrm{RhCl}-$ $\left.(\mathrm{CO})_{2}\right]_{2}$ 催化下溴代芳烃和吡啶可发生交叉偶联反应生 成 2-芳基吡啶, $0 \sim 86 \%$ 产率(Eq. 21).

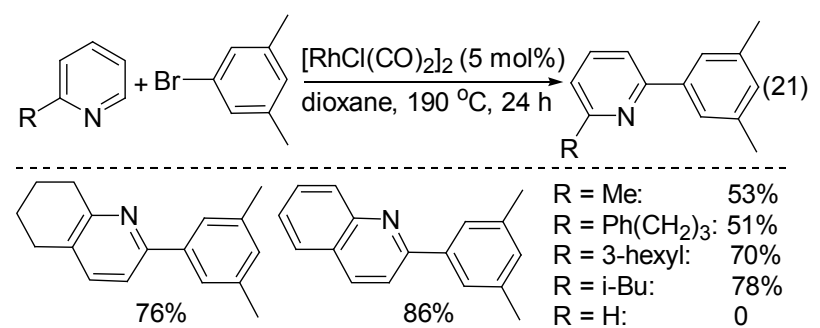

2008 年美国休斯敦大学的 Daugulis 等 ${ }^{[29]}$ 报道了在 $\mathrm{CuI} /$ Phenanthroline 催化下氧化吡啶和碘代芳烃可发生 交叉偶联反应生成 2-芳基基氧化吡定，41\%～91\%产率 (Eq. 22).

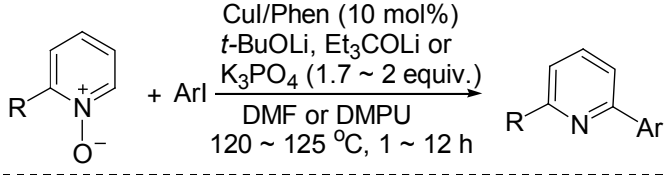

$$
\begin{aligned}
& \mathrm{R}=\mathrm{H}, \quad \mathrm{Ar}=\mathrm{Ph}: \quad 58 \% \\
& \mathrm{R}=\mathrm{H}, \quad \mathrm{Ar}=2-\mathrm{Py}: \quad 41 \% \\
& \mathrm{R}=\mathrm{Me}, \mathrm{Ar}=\mathrm{Ph}: \quad 43 \% \\
& \mathrm{R}=\mathrm{Ph}, \mathrm{Ar}=4-\mathrm{CF}_{3} \mathrm{C}_{6} \mathrm{H}_{4}: 80 \% \\
& \mathrm{R}=\mathrm{Ph}, \mathrm{Ar}=1 \text {-Naphthyl: } 91 \%
\end{aligned}
$$

2010 年四川大学的胡常伟和余孝其等 ${ }^{[30]}$ 报道了铁 催化的芳基硼酸和吡啶的偶联反应，主要生成 2-芳基吡 啶, 26\% 42\%产率(Eq. 23).
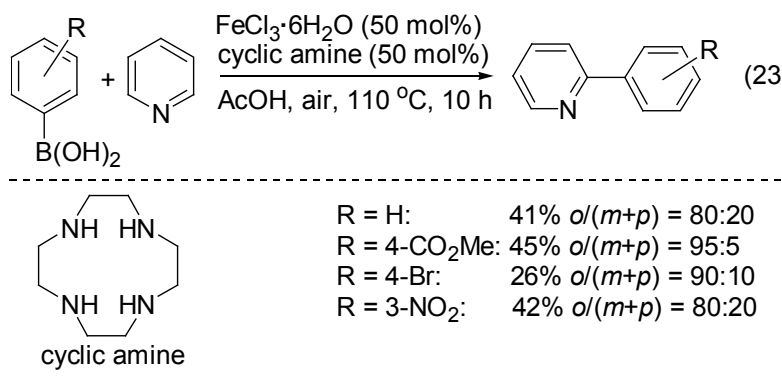
2009 年清华大学的华瑞茂等 ${ }^{[31]}$ 报道了金催化的吡 啶与溴代芳烃的交叉偶联反应，生成 2, 3, 4 位芳基取代 的吡定, 产率较低, 没有明显的区域选择性(Eq. 24).

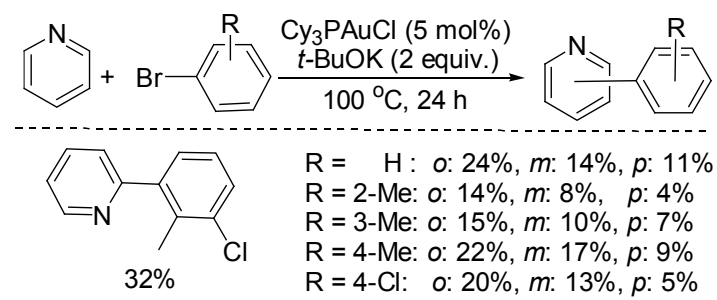

2010 年美国 Scripps 研究所的 Baran 等 ${ }^{[32]}$ 报道了 $\mathrm{AgNO}_{3}$ 催化下苯硣酸与吡啶发生交叉偶联反应, 主要 生成 2-芳基吡啶，产率 21\%～72\%(Eq. 25).

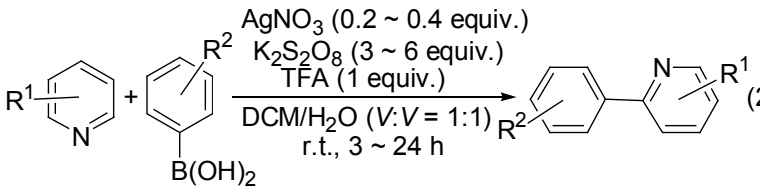

$$
\begin{aligned}
& \mathrm{R}^{1}=4-t-\mathrm{Bu} \\
& \mathrm{R}^{2}=\mathrm{H}: \quad 57 \%, \mathrm{R}^{2}=4-\mathrm{Br}: \quad 44 \% \\
& \mathrm{R}^{2}=2 \text {-OMe: } 33 \%, \mathrm{R}^{2}=3-\mathrm{Br}: \quad 53 \% \\
& \mathrm{R}^{2}=2 \text {-Me: } \quad 21 \%, \mathrm{R}^{2}=4-\mathrm{OPh}: \quad 72 \% \\
& \mathrm{R}^{2}=3-\mathrm{Me}: \quad 50 \%, \mathrm{R}^{2}=4-\mathrm{OEt}-3-\mathrm{Cl}: 58 \% \\
& \mathrm{R}^{2}=4-\mathrm{Me}: \quad 60 \%, \mathrm{R}^{2}=3-\mathrm{Ph}: \quad 60 \% \\
& \mathrm{R}^{2}=3,4-\mathrm{Me}_{2}: 58 \%, \mathrm{R}^{2}=4-\mathrm{CO}_{2} \mathrm{Me}: \quad 61 \% \\
& \mathrm{R}^{2}=4-\mathrm{F}: \quad 52 \%, \mathrm{R}^{2}=3-\mathrm{CN}: \quad 47 \% \\
& \mathrm{R}^{2}=4-\mathrm{I}: \quad 42 \%, \mathrm{R}^{2}=4-\mathrm{NO}_{2}: \quad 45 \% \\
& \mathrm{R}^{2}=4-\mathrm{CF}_{3}: \quad 37 \% \\
& R^{2}=4-M e \\
& \mathrm{R}^{1}=4-\mathrm{CF}_{3}: 81 \% \\
& \mathrm{R}^{1}=4-\mathrm{CN}: 92 \%(\mathrm{C} 2 / \mathrm{C} 3=2.7: 1)
\end{aligned}
$$

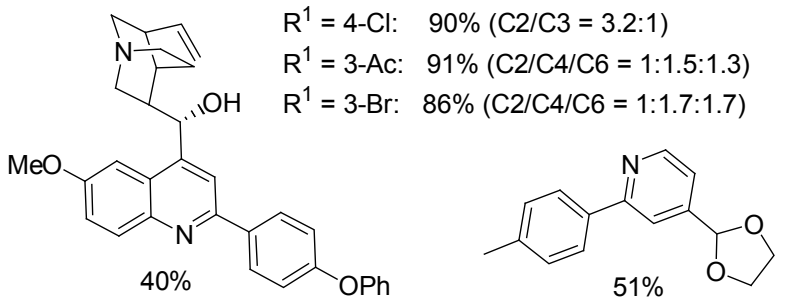

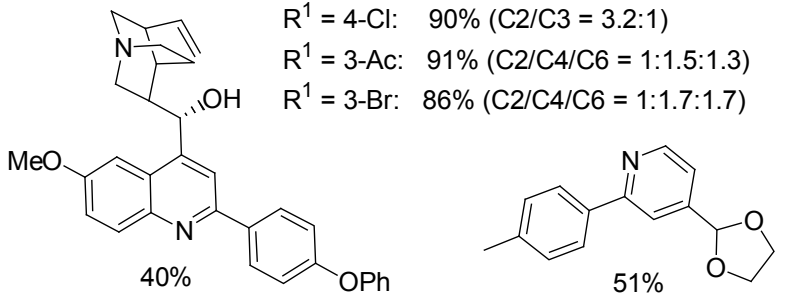

2005 年美国哥伦比亚大学的 Sames 等 ${ }^{[33]}$ 报道了在 钉催化剂作用下吡啶和碘苯反应生成 2-苯基吡定，70\% 产率.

2007 年日本东京技术研究所的 Suzuki 等 ${ }^{[34]}$ 报道了 在双钉催化剂作用下对位取代的吡啶脱氢自偶联生成 2,2'-联吡啶，产率 20\% 64\% (Eq. 26).

反应机理如 Scheme 16 所示: 双钓催化剂与吡啶配 位脱去一分子苯生成 $\mathbf{A}, \mathbf{A}$ 与吡啶作用脱去一分子 $\mathrm{H}_{2}$ 生 成 $\mathbf{B}$ 和产物.

\section{4 吡啶 2 位上发生的其它反应}

1992 年美国阿莫科化学公司的 Moore 等 ${ }^{[35]}$ 报道了
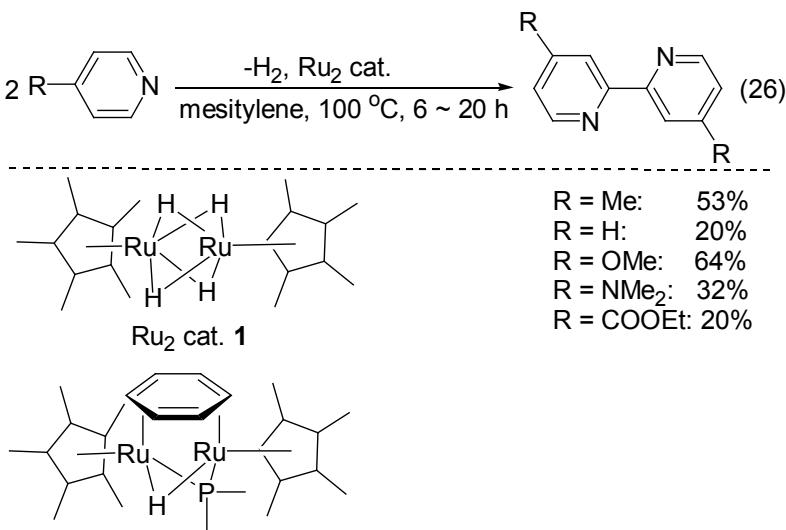

$\mathrm{Ru}_{2}$ cat. 2

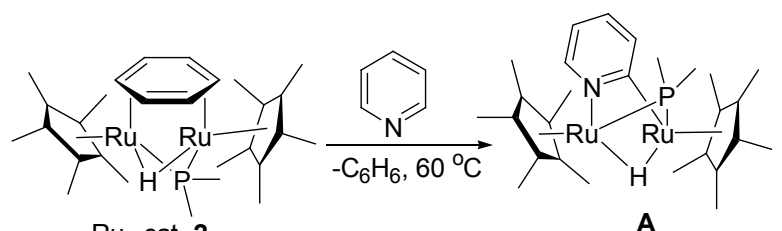

$\mathrm{Ru}_{2}$ cat. 2

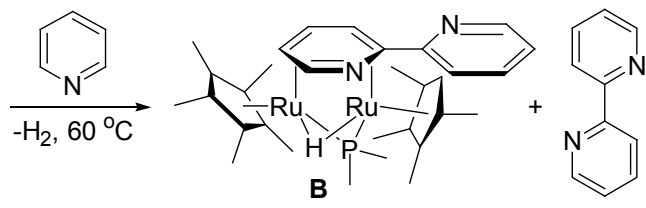

Scheme 16

在钉催化剂作用下吡啶 2 位上的羰基化，直链羰基化产 物 35\%，支链羰基化产物 29\% (Eq. 27).

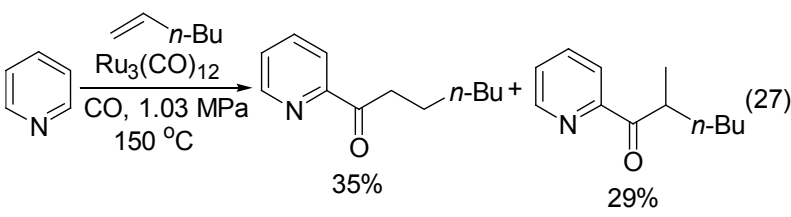

2006 年西班牙的 Esteruelas 等 ${ }^{[36]}$ 报道了喹啉的 2 位 能被 Os 和 $\mathrm{Ru}$ 活化, 形成金属配合物(Scheme 17).

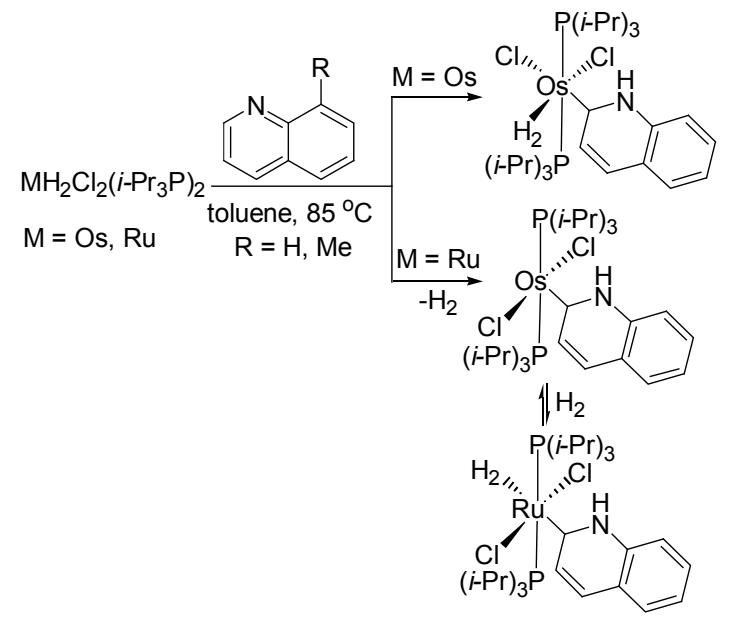

Scheme 17

Chin. J. Org. Chem. 2012, 32, 1653 1665 
2006 年西班牙的 Poveda 和 Carmona 等 ${ }^{[37]}$ 报道了 Ir 活化 2 位取代的吡啶形成氮杂卡宾(Eq. 28).

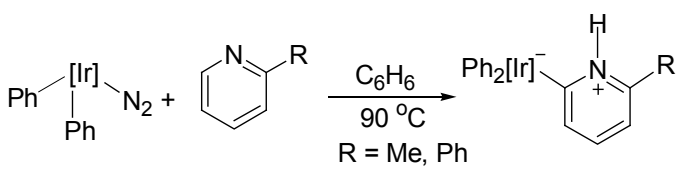

2011 年李朝军等 ${ }^{[38]}$ 报道了在钯催化下和过氧化合 物引发下含氮杂环化合物和简单醇发生脱氢交叉偶联 反应(Eq. 29).

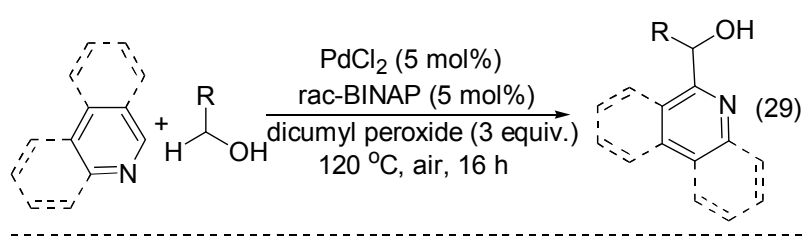

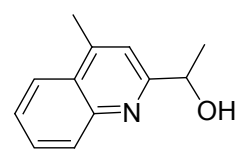

$71 \%$<smiles>[R]C(O)c1nc2ccccc2c2ccccc12</smiles>

$74 \%$<smiles>Cc1cc2ccccc2c(C(C)O)n1</smiles>

$64 \%$<smiles>CC(O)c1cc(C(C)O)c2ccccc2n1</smiles>

$62 \%(\mathrm{C} 2: \mathrm{C} 4:$ bis $=38: 10: 14)$
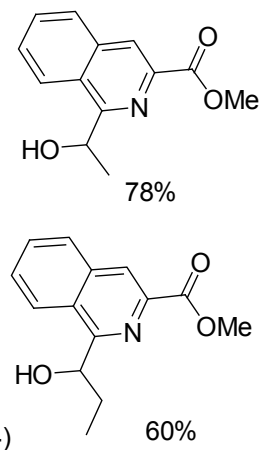

\section{2 吡啶 3 位上发生的官能化反应}

2002 年日本北海道大学的 Miyaura 等 ${ }^{[39]}$ 报道了吡 啶在 $[\mathrm{IrCl}(\mathrm{cod})]_{2}$ 催化下吡啶的间位嗍基化和间位双嗍基 化(Eq. 30).

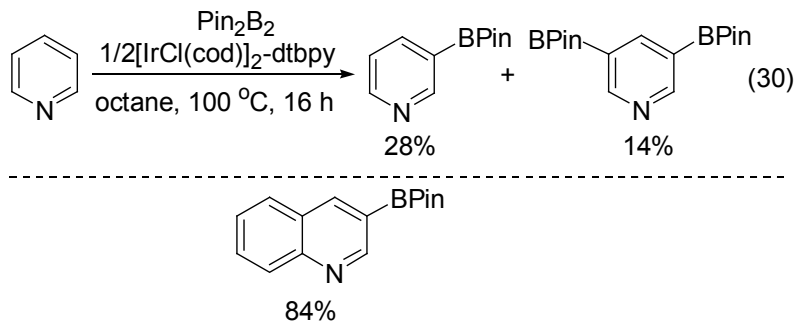

2010 年加拿大皇后大学的 Snieckus 等 ${ }^{[40]}$ 报道了 $[\operatorname{Ir}(\mathrm{cod})(\mathrm{OMe})]_{2}$ 催化下吡啶的间位嗍基化(Eq. 31).

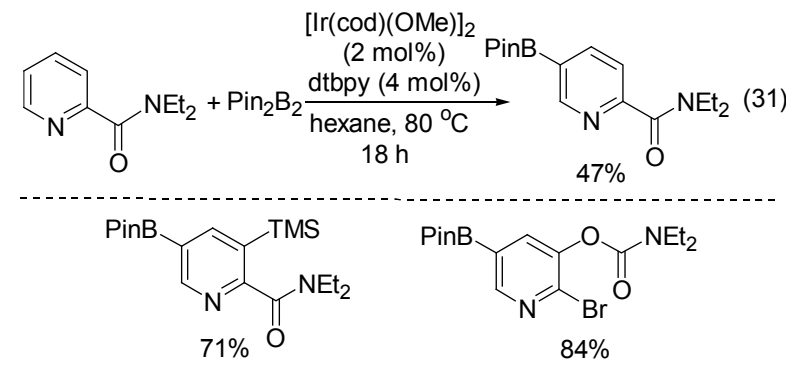

2007 年美国伊利诺伊大学香槟分校的 Hartwig 等 ${ }^{[41]}$
报道了 $[\operatorname{Ir}(\operatorname{cod})(\mathrm{OMe})]_{2}$ 催化下吡定的间位卤化，经历的 中间体是吡啶间位硣基化产物(Scheme 18).

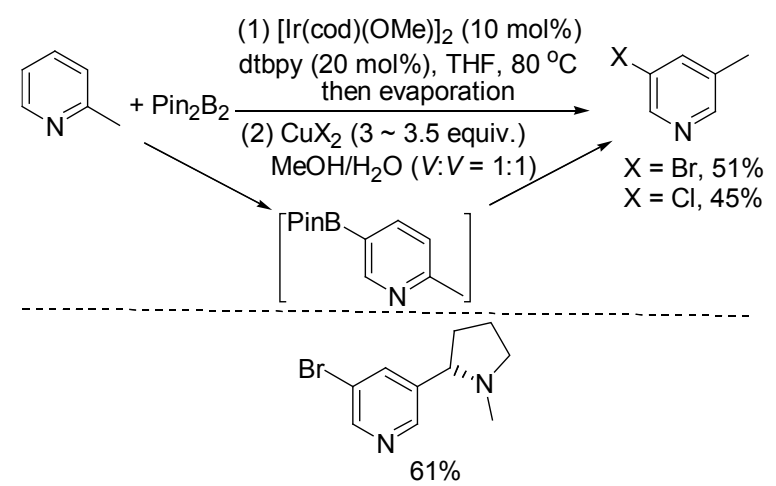

Scheme 18

2009 年意大利米兰大学的 Beccalli 等 ${ }^{[42]}$ 报道了 $\mathrm{Pd}(\mathrm{OAc})_{2}$ 催化的分子内的间位芳基化(Eq. 32).

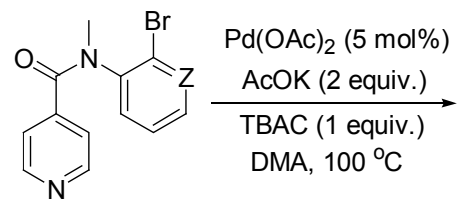<smiles>C=[Z]1cccc2c1c1cnccc1c(=O)n2C</smiles>

$\mathrm{Z}=\mathrm{CH}, \mathrm{N}: 58 \% \sim 69 \%$
2010 年美国 Scripps 研究所的余金权等 ${ }^{[1]}$ 报道了 $\mathrm{Pd}(\mathrm{OAc})_{2}$ 催化下尼古丁衍生物的间位芳基化，62\% 94\%产率(Eq. 33).

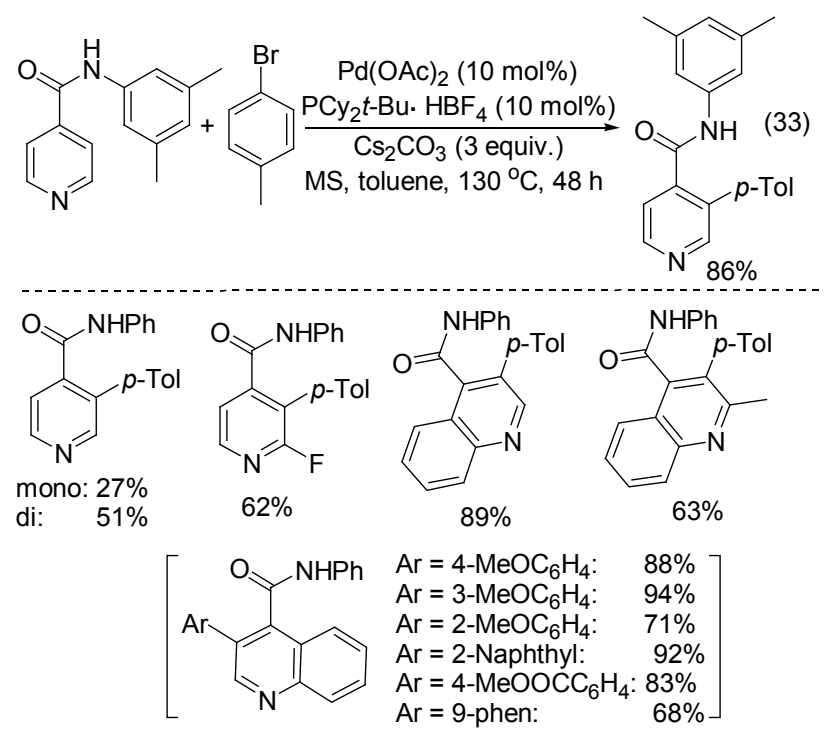

2011 年余金权等 ${ }^{[43]}$ 报道了 $\mathrm{Pd}(\mathrm{OAc})_{2}$ 催化的吡啶的 间位芳基化，具有很高的区域选择性，产物主要是间位 的，邻位和对位芳基化较少; 产率 54\% 90\% (Eq. 34).

可能的反应机理如 Scheme 19 所示: $\mathrm{Pd}(\mathrm{OAc})_{2}$ 夺取 吡啶 3 位的氢脱去一分子 HOAc 生成 $\mathbf{A}, \mathbf{A}$ 与 $\mathrm{PhI}$ 氧化 加成生成 $\mathbf{B}, \mathbf{B}$ 还原消除生成产物. 

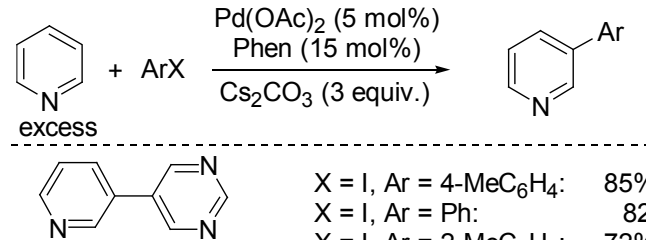

$\mathrm{X}=\mathrm{I}, \mathrm{Ar}=4-\mathrm{MeC}_{6} \mathrm{H}_{4}: \quad 85 \%$ $\mathrm{X}=\mathrm{I}, \mathrm{Ar}=\mathrm{Ph}: \quad 82 \%$ $\mathrm{X}=\mathrm{I}, \mathrm{Ar}=2-\mathrm{MeC}_{6} \mathrm{H}_{4}: \quad 72 \%$ $\begin{array}{ll}X=\mathrm{Br}, 54 \% & \mathrm{X}=\mathrm{I}, \mathrm{Ar}=4-\mathrm{MeOC}_{6} \mathrm{H}_{4}: 72 \% \\ & \mathrm{X}=\mathrm{I}, \mathrm{Ar}=4-\mathrm{FC}_{6} \mathrm{H}_{4}: \quad 66 \%\end{array}$

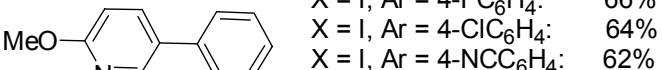
$\mathrm{X}=\mathrm{I}, \mathrm{Ar}=4-\mathrm{CF}_{3} \mathrm{C}_{6} \mathrm{H}_{4}: \quad 75 \%$ $\mathrm{X}=\mathrm{I}, \mathrm{Ar}=3-\mathrm{CF}_{3} \mathrm{C}_{6} \mathrm{H}_{4}: \quad 72 \%$ $\mathrm{X}=\mathrm{I}, \mathrm{Ar}=2-\mathrm{CF}_{3} \mathrm{C}_{6} \mathrm{H}_{4}: \quad 80 \%$ $\mathrm{X}=\mathrm{I}, \mathrm{Ar}=4-\mathrm{EtO}_{2} \mathrm{CC}_{6} \mathrm{H}_{4}: 56 \%$ $\mathrm{X}=\mathrm{Br}, \mathrm{Ar}=2,5-\mathrm{Me}_{2} \mathrm{C}_{6} \mathrm{H}_{4}: 79 \%$ $\mathrm{X}=\mathrm{Br}, \mathrm{Ar}=4-\mathrm{PhC}_{6} \mathrm{H}_{4}: \quad 73 \%$ $\mathrm{X}=\mathrm{Br}, \mathrm{Ar}=2-$ Naphthyl: $\quad 73 \%$ $\mathrm{X}=\mathrm{Br}, \mathrm{Ar}=4-\mathrm{MeSC}_{6} \mathrm{H}_{4}: \quad 86 \%$ $X=I, 65 \%$ $\mathrm{X}=\mathrm{Br}, \mathrm{Ar}=3-\mathrm{Py}$ :

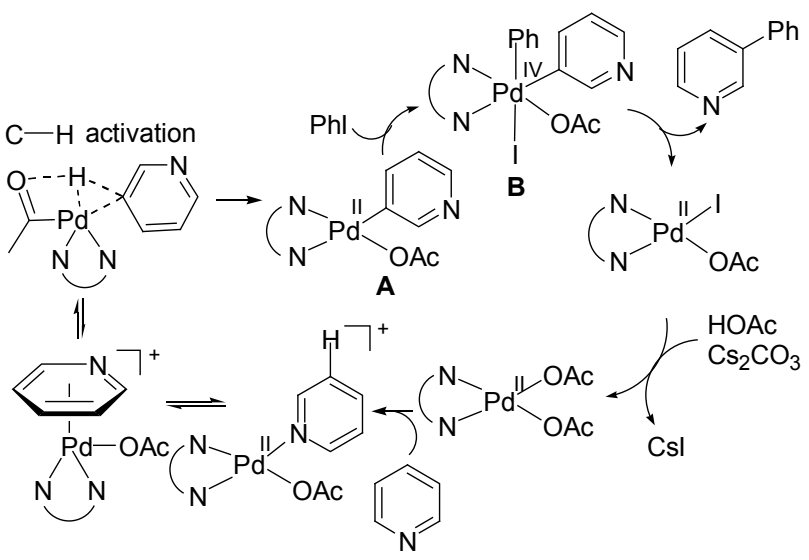

Scheme 19

2011 年余金权等 ${ }^{[44]}$ 报道了 $\mathrm{Pd}(\mathrm{OAc})_{2}$ 催化的吡定的 间位烯基化，具有很高的区域选择性，产物主要是间位 的, 邻位和对位芳基化较少(Eq. 35). 可能的反应机理和 Scheme 19 类似, 所不同的是本反应还原消除得到的 $\mathrm{Pd}(0)$ 被 $\mathrm{Ag}_{2} \mathrm{CO}_{3}$ 氧化成二价钯, 完成催化循环.

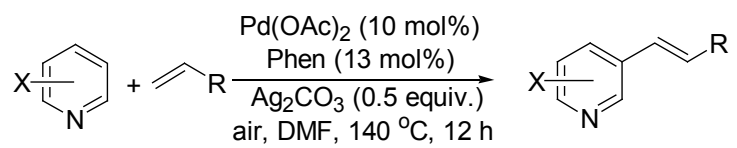

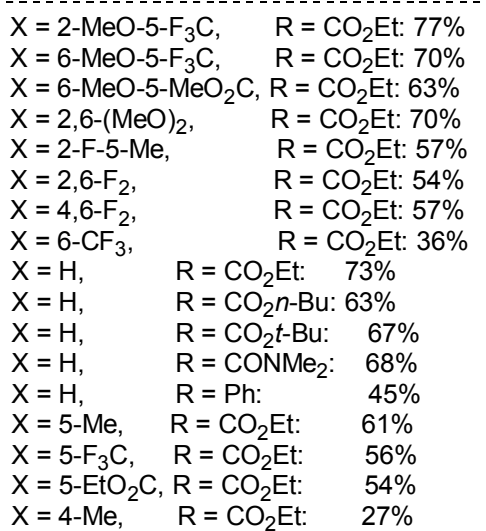

2011 年北京大学的施章杰等 ${ }^{[45}$ 报道了 $\operatorname{Ir}_{4}(\mathrm{CO})_{12}$ 催 化下吡啶间位的官能化. 吡啶经 Ir 碳氢键活化的中间体 对醛加成(Eq. 36).

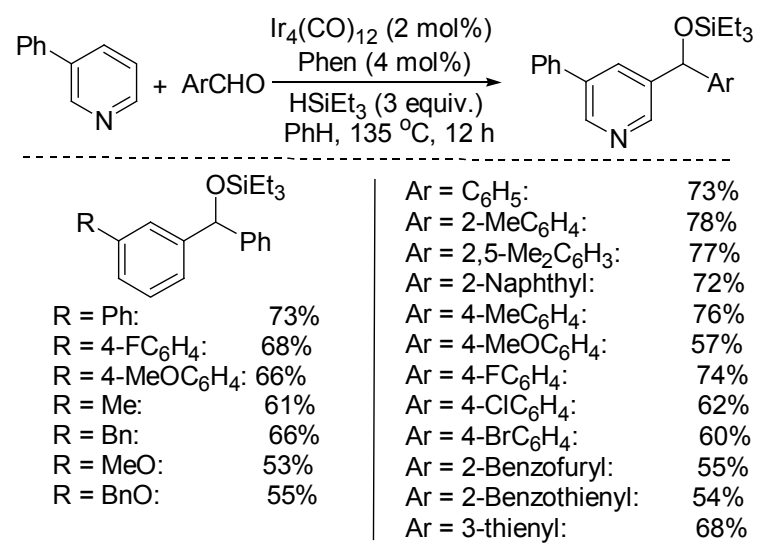

反应机理如 Scheme 20 所示: $\mathrm{Ir}_{4}(\mathrm{CO})_{12}$ 和 $\mathrm{HSiEt}_{3}$ 形 成硅铱中间体 $\mathbf{A}, \mathbf{A}$ 使吡定间位的 $\mathrm{C}-\mathrm{H}$ 键活化, 生成 $\mathbf{B}, \mathbf{B}$ 亲氧，能对醛基加成生成 $\mathbf{C}, \mathbf{C}$ 还原消除得到产物 和铱氢化物 $\mathbf{D}, \mathbf{D}$ 经 $\mathrm{HSiEt}_{3}$ 氧化生成硅铱中间体 $\mathbf{A}$, 完 成了催化循环.

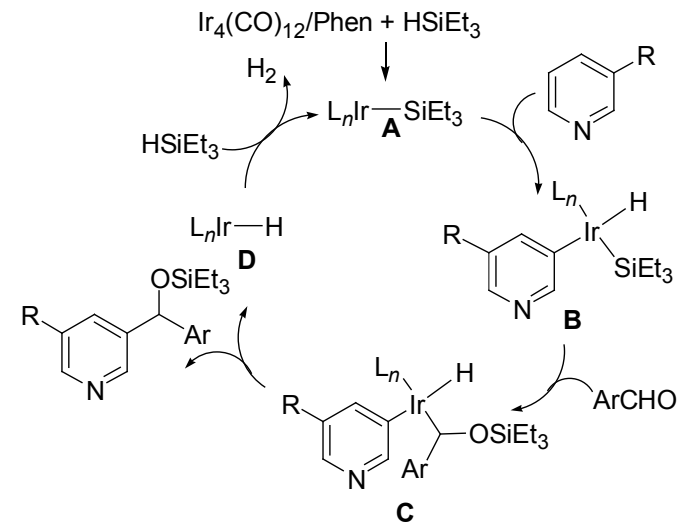

Scheme 20

1997 年英国利兹大学的 Grigg 等 ${ }^{[46]}$ 报道了对位乙酰 化的吡啶在钉催化下发生间位的烷基化(Eq. 37).

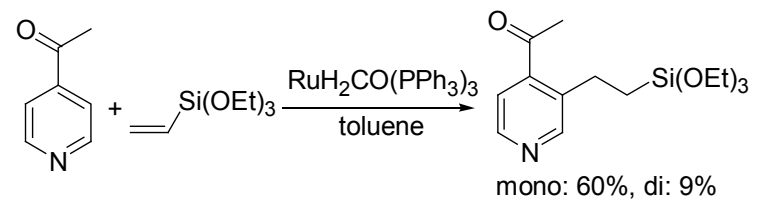

\section{3 吡啶 4 位上发生的官能化反应}

2006 年英国杜伦大学的 Marder 等 ${ }^{[47]}$ 报道了 $[\operatorname{Ir}(\mathrm{cod})(\mathrm{OMe})]_{2}$ 可使吡啶的对位和间位发生硼基化.

2007 年 Hartwig 等 ${ }^{[41]}$ 报道了 $[\operatorname{Ir}(\mathrm{cod})(\mathrm{OMe})]_{2}$ 催化下 吡啶的对位卤化，经历的中间体是吡啶对位嗍基化产物 (Scheme 21). 


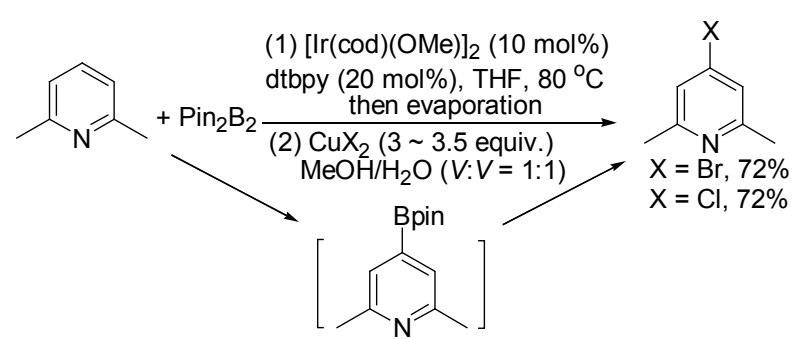

Scheme 21

2012 年 Hartwig 等 ${ }^{[48]}$ 报道了 $[\operatorname{Ir}(\operatorname{cod})(\mathrm{OMe})]_{2}$ 催化下 吡啶的对位三氟甲基化, 经历的中间体是吡啶对位硼基 化产物(Eq. 38).

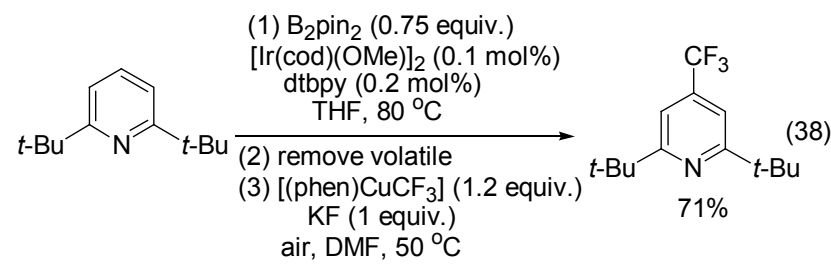

2000 年密西根州立大学的 Smith III 等 ${ }^{[49]}$ 报道了 $\mathrm{Cp} * \mathrm{Rh}\left(\eta^{4}-\mathrm{C}_{6} \mathrm{Me}_{6}\right)$ 催化的 2,6-二甲基吡啶的 4 位硼基化, 产率 $41 \%$.

1997 年 Grigg 等 ${ }^{[46]}$ 报道了间位乙酰化的吡定在钉催 化下发生对位的烷基化(Eq. 39).

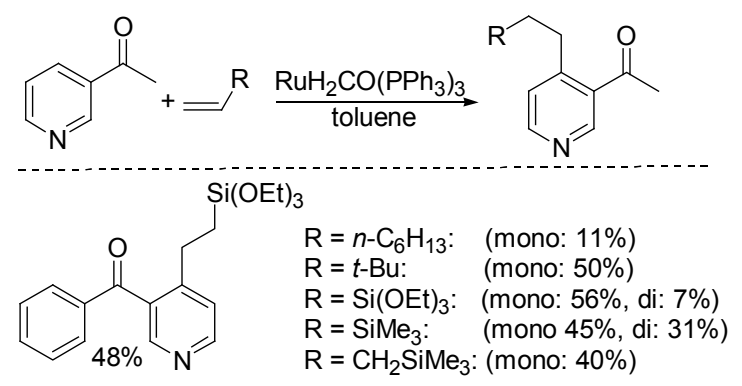

2010 年 Nakao 和 Hiyama 等 ${ }^{[50]}$ 报道了 $\mathrm{Ni}(\operatorname{cod})_{2}$ 催化 的吡啶对位烷基化, 主要生成直链烷基化产物, 只有吡 啶和苯乙烯反应主要生成支链烷基化产物(Eq. 40).

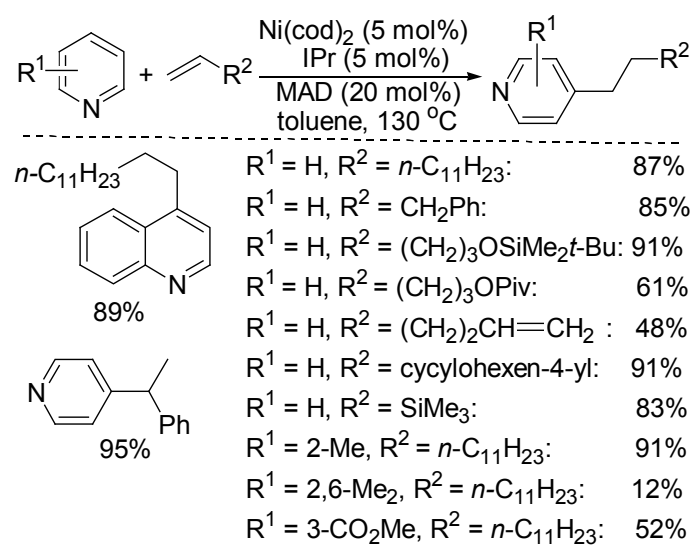

反应机理如 Scheme 21 所示: 零价镍与吡啶配位形 成 $\mathbf{A}$, 零价镍对吡啶的 4 位碳氢键活化生成 $\mathbf{B}, \mathbf{B}$ 与烯烃 配位生成 $\mathbf{C}, \mathbf{C}$ 对烯烃插入生成 $\mathbf{D}, \mathbf{D}$ 发生还原消除生成 产物.

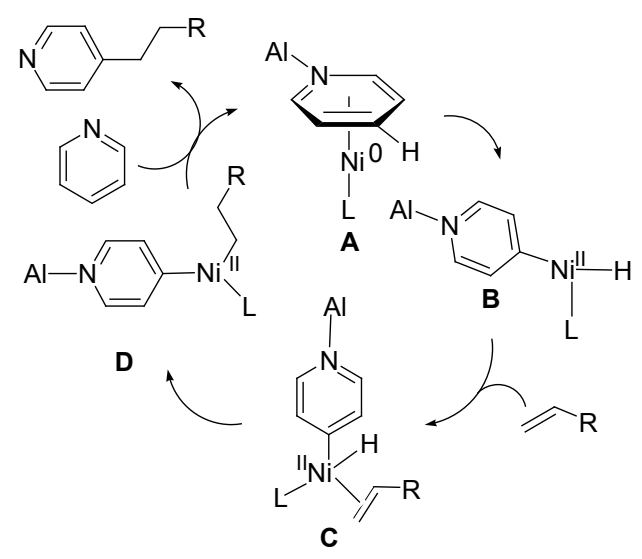

Scheme 21

该文还报道了在镍催化下吡啶能与 4-辛炔反应生 成对位烯基取代的吡啶, 产率 53\% ${ }^{[50]}$.

2010 年台湾化学所的 Ong 等 ${ }^{[51]}$ 报道了在 $\mathrm{Ni}(\operatorname{cod})_{2}$ 催化的吡啶的对位烯基化, 主要生成对位烯基化产物, 个别底物少部分生成间位烯基化产物, 在反应过程中未 观察到邻位烯基化的产物(Eq. 41). 反应机理与 Scheme 21 相似，用炔烃替代烯烃即可.

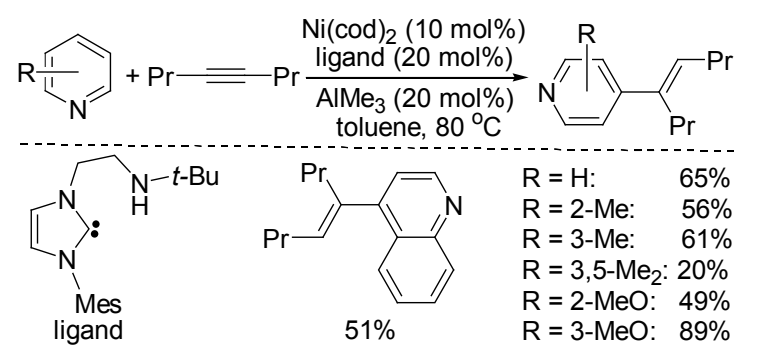

2010 年余金权等 ${ }^{[1]}$ 报道了 $\mathrm{Pd}(\mathrm{OAc})_{2}$ 催化下尼古丁 衍生物的对位芳基化, 44\% 60\%产率(Eq. 42).

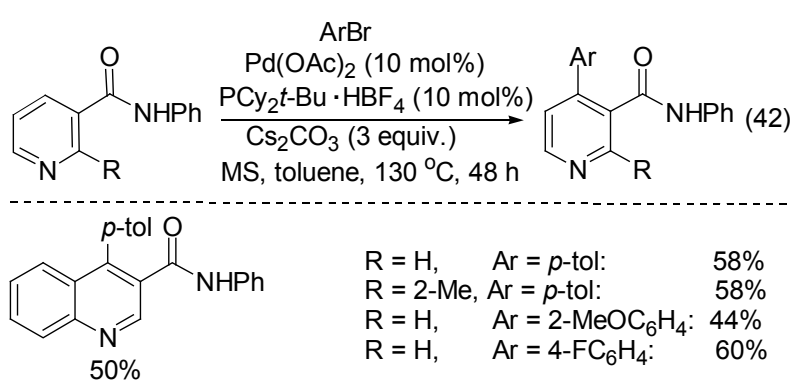

2011 年美国哥伦比亚大学的 Sames 等 ${ }^{[52]}$ 报道了在 $\mathrm{Pd}(\mathrm{OAc})_{2}$ 催化下间硝基吡啶发生对位的芳基化, 产率 $30 \% \sim 81 \%$ (Eq. 43). 间位有 $\mathrm{F}, \mathrm{Cl}, \mathrm{CN}$ 取代的吡啶在对 位都可发生芳基化. 


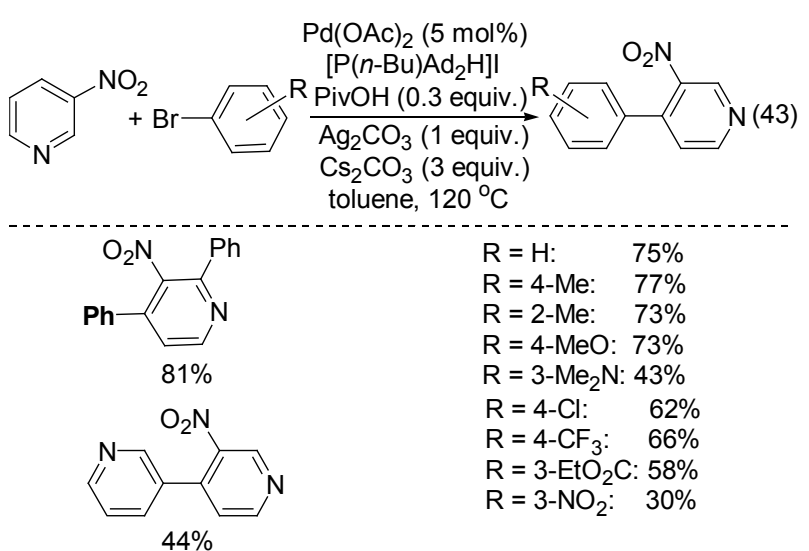

2011 年美国伊利诺伊大学芝加哥分校的 Driver 等 ${ }^{\left[{ }^{[3]}\right.}$ 报道了 3 -吡啶基取代的芳基叠氮化合物在钓催化 下在吡啶的对位发生关环反应，生成 $\gamma$-Carbolinium ion, 反应有很大的官能团兼容性, $\mathrm{R}^{1}$ 可以是吸电子基和供电 子基, $\mathrm{R}^{2}$ 如果是 $\mathrm{O}$ 则不发生反应(Eq. 44).

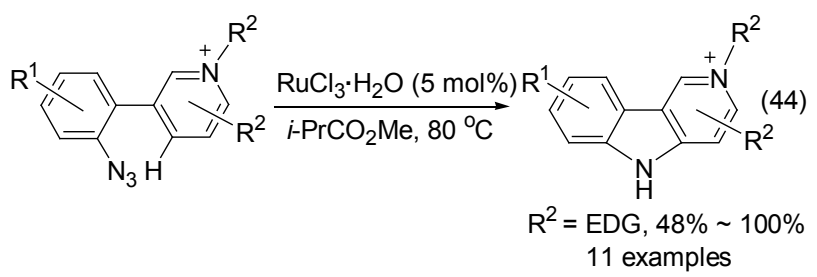

\section{4 吡啶的官能化在药物和天然产物全合成的应 用}

Complanadine A 能增强神经生长因子的 mRNA 表 达和增强人体神经胶质细胞中神经生长因子的产量. 2010 年美国加州大学伯克利分校的 Sarpong 等 ${ }^{[54]}$ 报道了 complanadine A 的全合成. 关键步骤是 $[\operatorname{Ir}(\operatorname{cod})(\mathrm{OMe})]_{2} /$ $\mathrm{d} t$-Bu-dipy 催化的吡啶的间位硼基化(Scheme 22). 关键 中间体获得了 $75 \%$ 产率.

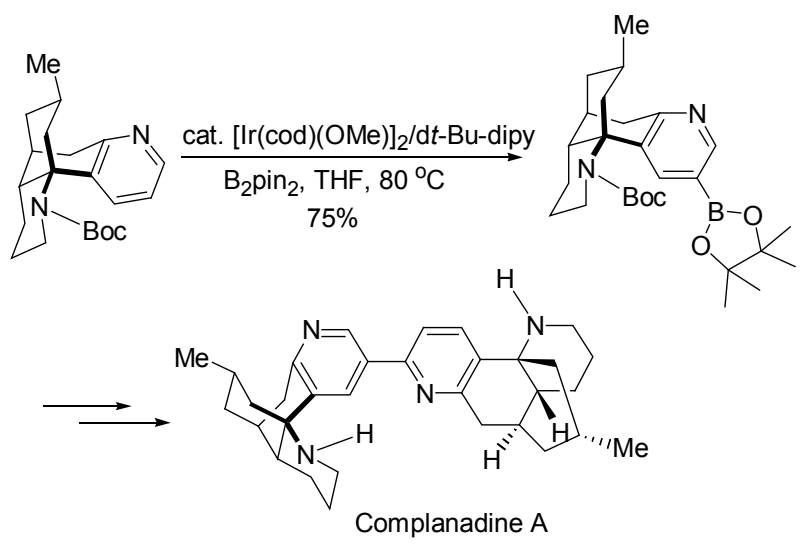

Scheme 22

Dimebolin 是一种能够阻止线粒体传导孔的形成的 胆碱酯酶抑制剂/NMDA 受体阻断剂, 是治疗阿尔茨海默
病的新药. 2011 年 Driver 等 ${ }^{[53]}$ 用他们开发的 $\mathrm{Ru}$ 催化的 吡啶的对位分子内关环反应用于 Dimebolin 的合成，关 键中间体获得了 91\%产率(Scheme 23).

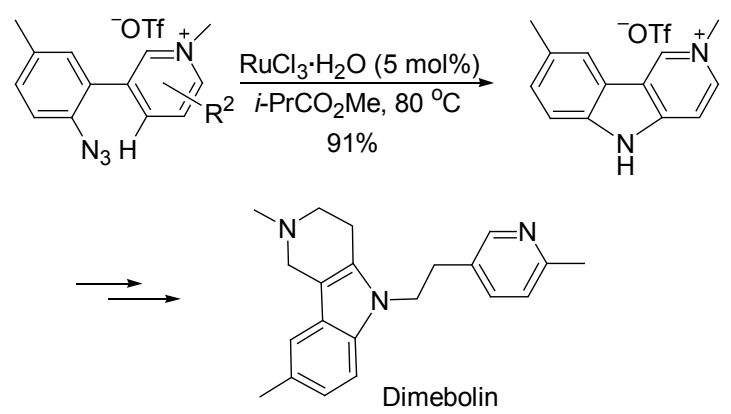

Scheme 23

Altinicline 用于治疗帕金森病. 最有效的从 (L)-nicotine 制备 Altinicline 的总产率是 17\%. 2007 年 Hartwig 等 ${ }^{[41]}$ 用他们开发的 $[\operatorname{Ir}(\operatorname{cod})(\mathrm{OMe})]_{2}$ 催化下吡啶 的间位卤化方法从 $(L)$-nicotine 制备 Altinicline 的总产率 是 54\% (Scheme 24). 关键中间体获得了 $61 \%$ 产率.

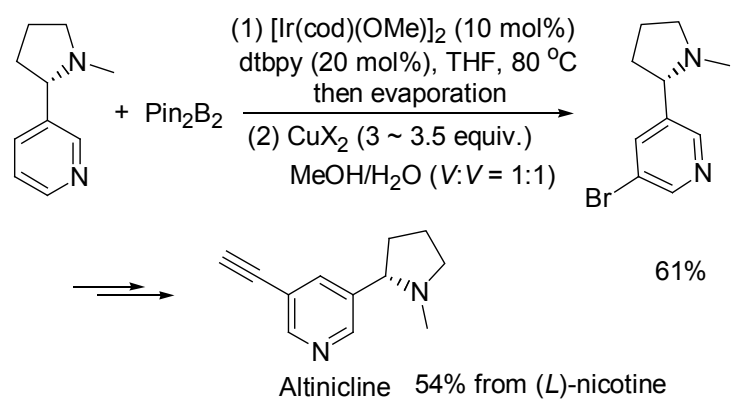

Scheme 24

Preclamol 是抗精神病药. 2011 年余金权等 ${ }^{[43]}$ 用他 们开发的 $\mathrm{Pd}(\mathrm{OAc})_{2}$ 催化的吡啶的间位芳基化用来合成 (土)-Preclamol，总产率 47\% (Scheme 25). 关键中间体 获得了 $70 \%$ 产率.

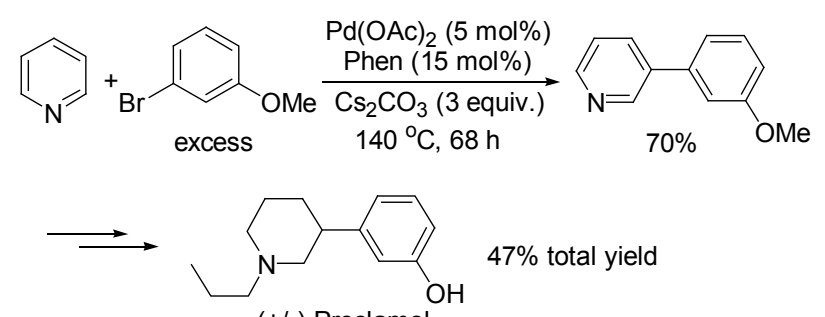

(+/-) Preclamol

Scheme 25

\section{5 结论}

总结了从 1985 年以来过渡金属催化的吡啶在 2 位、 3 位、4 位上发生的烷基化、烯基化、芳基化和酰基化 
等直接官能化反应. 过渡金属催化的吡啶的 $\mathrm{C}-\mathrm{H}$ 键活 化反应是构建 $\mathrm{C}-\mathrm{C}$ 键和 $\mathrm{C}-\mathrm{B}$ 键的有效手段, 能实现 常规反应之不能, 特别是在药物分子和天然产物的合成 中得到了有效的利用.

之前所报道的吡定的 $\mathrm{C}-\mathrm{H}$ 键的活化都是关于 $\mathrm{C}-$ $\mathrm{C}$ 键和 $\mathrm{C}-\mathrm{B}$ 键的构筑, 关于 $\mathrm{C}-\mathrm{O}$ 键、 $\mathrm{C}-\mathrm{S}$ 键、 $\mathrm{C}-\mathrm{N}$ 键等的构筑尚未见报道, 基于吡啶的 $\mathrm{C}-\mathrm{H}$ 键的活化还 有很多的工作可以研究.

\section{References}

[1] Wasa, M.; Worrell, B. T.; Yu, J. Angew. Chem., Int. Ed. 2010, 49, 1275 .

[2] Mousseau, J. J.; Bull, J. A.; Charette, A. B. Angew. Chem., Int. Ed. 2010, 49, 1115

[3] Vanderwal, C. D. J. Org. Chem. 2011, 76, 9555.

[4] Martin, D. B. C.; Nguyen, L. Q.; Vanderwal, C. D. J. Org. Chem. 2012, 77, 17.

[5] Nakao, Y. Synthesis 2011, 3209.

[6] Eisenstadt, A.; Giandomenico, C. M.; Frederick, M. F.; Laine, R. M. Organometallics 1985, 4, 2033.

[7] Jordan, R. F.; Taylor, D. F. J. Am. Chem. Soc. 1989, 111, 778.

[8] Rodewald, S. Jordan, R. F. J. Am. Chem. Soc. 1994, 116, 4491.

[9] Lewis, J. C.; Bergman, R. G.; Ellman, J. A. J. Am. Chem. Soc. 2007, 129, 5332.

[10] Yotphan, S.; Bergman, R. G.; Ellman, J. A. Org. Lett. 2010, 12, 2978.

[11] Deng, G.; Li, C. Org. Lett. 2009, 11, 1171.

[12] Guan, B.; Hou, Z. J. Am. Chem. Soc. 2011, 133, 18086.

[13] Murakami, M.; Hori, S. J. Am. Chem. Soc. 2003, 125, 4720.

[14] Kanyiva, K. S.; Nakao, Y.; Hiyama, T. Angew. Chem., Int. Ed. 2007, 46, 8872.

[15] Nakao, Y.; Kanyiva, K. S.; Hiyama, T. J. Am. Chem. Soc. 2008, 130, 2448-2449.

[16] Cho, S. H.; Hwang, S. J.; Chang, S. J. Am. Chem. Soc. 2008, 130, 9254.

[17] Wu, J.; Cui, X.; Chen, L.; Jiang, G.; Wu, Y. J. Am. Chem. Soc. 2009, 131, 13888 .

[18] Song, G.; Gong, X.; Li, X. J. Org. Chem. 2011, 76, 7583.

[19] Mukhopadhyay, S.; Rothenberg, G.; Gitis, D.; Baidossi, M.; Ponde, D. E.; Sasson, Y. J. Chem. Soc., Perkin Trans. 2 2000, 1809.

[20] Campeau, L.; Rousseaux, S.; Fagnou, K. J. Am. Chem. Soc. 2005, $127,18020$.

[21] Larivée, A.; Mousseau, J. J.; Charette, A. B. J. Am. Chem. Soc. 2008, 130, 52.

[22] Xi, P.; Yang, F.; Qin, S.; Zhao, D.; Lan, J.; Gao, G.; Hu, C.; You, J. J. Am. Chem. Soc. 2010, 132, 1822.

[23] Gong, X.; Song, G.; Zhang, H.; Li, X. Org. Lett. 2011, 13, 1766.

[24] Duric, S.; Tzschucke, C. C. Org. Lett. 2011, 13, 2310.

[25] Tan, Y.; Barrios-Landeros, F.; Hartwig, J. F. J. Am. Chem. Soc. 2012, 134, 3683.
[26] Tobisu, M.; Hyodo, I.; Chatani, N. J. Am. Chem. Soc. 2009, 131, 12070 .

[27] Kobayashi, O.; Uraguchi, D.; Yamakawa, T. Org. Lett. 2009, 11, 2679.

[28] Berman, A. M.; Lewis, J. C.; Bergman, R. G.; Ellman, J. A. J. Am. Chem. Soc. 2008, 130, 14926.

[29] Do, H.; Khan, R. M. K.; Daugulis, O. J. Am. Chem. Soc. 2008, 130, 15185 .

[30] Wen, J.; Qin, S.; Ma, L.; Dong, L.; Zhang, J.; Liu, S.; Duan, Y.; Chen, S.; Hu, C.; Yu, X. Org. Lett. 2010, 12, 2694.

[31] Li, M.; Hua, R. Tetrahedron Lett. 2009, 50, 1478.

[32] Seiple, I. B.; Su, S.; Rodriguez, R. A.; Gianatassio, R.; Fujiwara, Y.; Sobel, A. L.; Baran, P. S. J. Am. Chem. Soc. 2010, 132, 13194.

[33] Godula, K.; Sezen, B.; Sames, D. J. Am. Chem. Soc. 2005, 127, 3648.

[34] Kawashima, T.; Takao, T.; Suzuki, H. J. Am. Chem. Soc. 2007, 129, 11006.

[35] Moore, E. J.; Pretzer, W. R.; O'Connell, T. J.; Harris, J.; LaBounty, L.; Chou, L.; Grimmer, S. S. J. Am. Chem. Soc. 1992, 114, 5888.

[36] Esteruelas, M. A.; Fernández-Alvarez, F. J.; Oñate, E. J. Am. Chem. Soc. 2006, 128, 13044.

[37] Alvarez, E.; Conejero, S.; Paneque, M.; Petronilho, A.; Poveda, M. L.; Serrano, O.; Carmona, E. J. Am. Chem. Soc. 2006, 128, 13060.

[38] Correia, C. A.; Yang, L.; Li, C. Org. Lett. 2011, 13, 4581.

[39] Takagi, J.; Sato, K.; Hartwig, J. F.; Ishiyamaa, T.; Miyaura, N. Tetrahedron Lett. 2002, 43, 5649.

[40] Hurst, T. E.; Macklin, T. K.; Becker, M.; Hartmann, E.; Kügel, W.; Salle, J. P.; Batsanov, A. S.; Marder, T. B.; Snieckus, V. Chem. Eur. J. 2010, 16, 8155 .

[41] Murphy, J. M.; Liao, X.; Hartwig, J. F. J. Am. Chem. Soc. 2007, 129, 15434.

[42] Basolo, L.; Beccalli, E. M.; Borsini, E.; Broggini, G. Tetrahedron 2009, 65, 3486.

[43] Ye, M.; Gao, G.; Edmunds, A. J. F.; Worthington, P. A.; Morris, J. A.; Yu, J. J. Am. Chem. Soc. 2011, 133, 19090.

[44] Ye, M.; Gao, G.; Yu, J. J. Am. Chem. Soc. 2011, 133, 6964.

[45] Li, B.; Shi, Z. Chem. Sci. 2011, 2, 488.

[46] Grigg, R.; Savic, V. Tetrahedron Lett. 1997, 38, 5737.

[47] Mkhalid, I. A. I.; Coventry, D. N.; Albesa-Jove, D.; Batsanov, A. S.; Howard, J. A. K.; Perutz, R. N.; Marder, T. B. Angew. Chem., Int. Ed. 2006, 45, 489 .

[48] Litvinas, N. D.; Fier, P. S.; Hartwig, J. F. Angew. Chem., Int. Ed. 2012, 51, 536.

[49] Cho, J.; Iverson, C. N.; Smith, III. M. R. J. Am. Chem. Soc. 2000, $122,12868$.

[50] Nakao, Y.; Yamada, Y.; Kashihara, N.; Hiyama, T. J. Am. Chem. Soc. 2010, 132, 13666

[51] Tsai, C.; Shih, W.; Fang, C.; Li, C.; Ong, T.; Yap, G. P. A. J. Am. Chem. Soc. 2010, 132, 11887.

[52] Guo, P.; Joo, J.; Rakshit, S.; Sames, D. J. Am. Chem. Soc. 2011, 133, 16338.

[53] Dong, H.; Latka, R. T.; Driver, T. G. Org. Lett. 2011, 13, 2726.

[54] Fisher, D. F.; Sarpong, R. J. Am. Chem. Soc. 2010, 132, 5926. 